\title{
آلية لتوطين الخدمات العامة في مصر بالاعتماد على توافقها
}

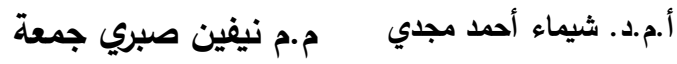

$$
\begin{aligned}
& \text { مدرس مساعد بقسم الهندسة }
\end{aligned}
$$

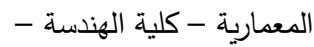

$$
\begin{aligned}
& \text { جامعة الفيوم }
\end{aligned}
$$

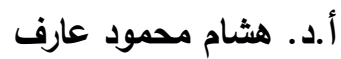

أستاذ التخطيط والتصميم

العراني

قسم الهندسة المعمارية-كلية

الهندسة - جامعة الفيوم

nsg00@fayoum.edu.eg Sam06@fayoum.edu.eg Hma00@fayoum.edu.eg

التوافق والوصول لمصفوفة التوافق بين الخدمات؛ كأداة

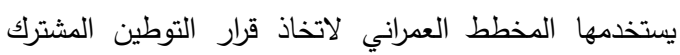
للخدمات، وتم اتباع المنهجية التالية:

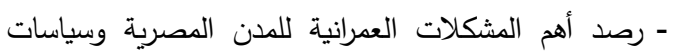

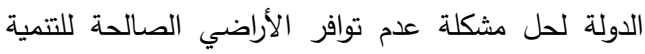

$$
\text { العمرانية (المنهج الوصفي). }
$$

- تحليل أدبيات تطبيق استخدامات الأراضي المختلطة والتوافق بين استخدامات الأراضي لتحديد متغيرات حساب درجة التابت الترات التوافق بين الخدمات في مصر . ( المنهج الوصفي والتحليلي). - إعداد مصفوفتي التوافق والتشارك بين الخدمات في مصر باستخدام المنهج التحليلي والاستتتاجي.

وتم التوصل لمصفوفة التوافق بين الخدمات في مصر لتساعد

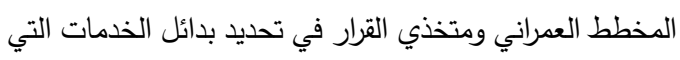

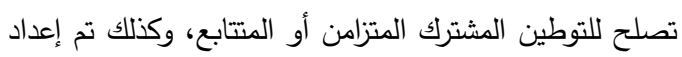

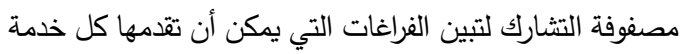

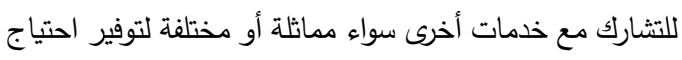
المدن الحالي والمستقبلي من الخدمات في إطار الاستخدام

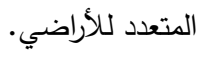

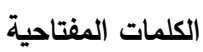

استخدامات الأراضي المختلطة - استخدامات الأراضي المتعددة - التوافق - توطين الخدمات الارطي النغات
ظهر مفهوم استخدامات الأراضي المختلطة كنتيجة

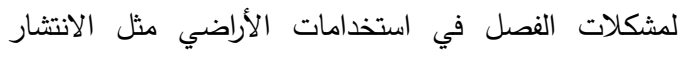

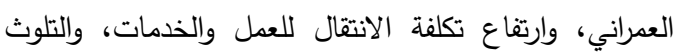

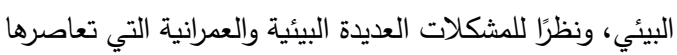

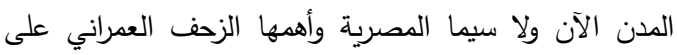

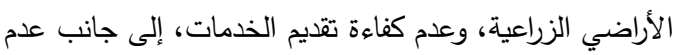

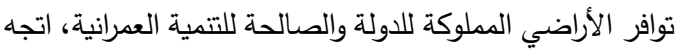
المخططون العمرانيون بالبحث لاقتراح حلول لهذه المشكلات، الهواته

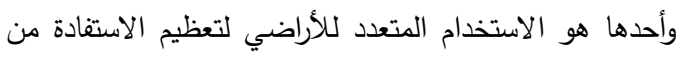

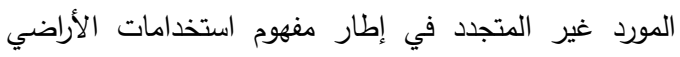

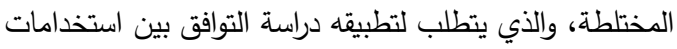

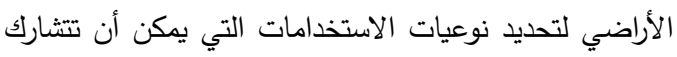
قطعة الأرض، ونمط هذا التشارك بالتزامن أو التتابع.

ترتكز إثكالية البحث على عدم وجود آلية حالية يتم

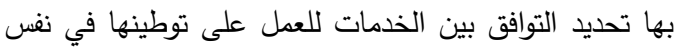

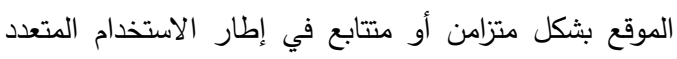

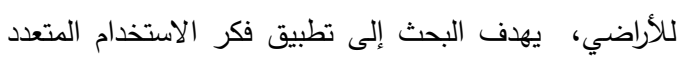

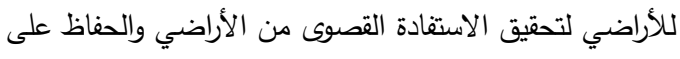

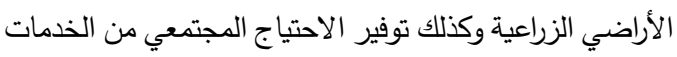

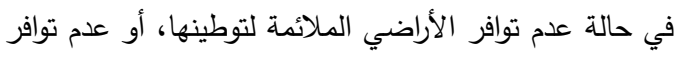

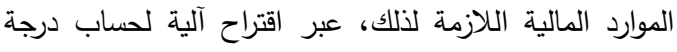


اختفاء الفراغات الخضراء وأصبحت المدن أشبه بكتلة

بنائية ضخمة صماء. [3] عدم كفاية الخدمات العامة وزيادة الضغط على المتوفر منها مقارنةً بالطاقة الاستيعابية لها بسبب الزيادة الكبيرة

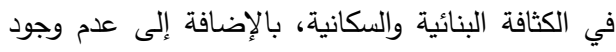

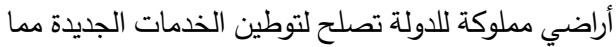

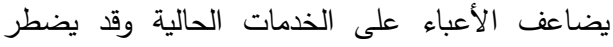
السكان إلى قطع رحلة طويلة للوصول إلى هذه الخدمات

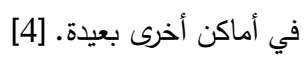

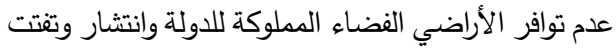
الملكيات الخاصة مما أدى لصعوبة توطين الخدمات المقترحة في المخططات الاستراتيجية العامة و التفصيلية.

[3] 2-1 سياسات الدولة لحل مشكلة عدم توافر الأراضي الصالحة للتنمية العمرانية تبنت هيئة التخطيط العمراني والمحليات عدة مقترحات للتغلب على مشكلة عدم توافر الأراضي اللازمة لتنفيذ المخططات الاستراتيجية والتنصيلية للمدن ومنها:

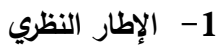

يتم فيه تناول أهم مشكلات العمران المصري ومراجعة أدبيات تطبيق الاستخدام المختلط للأراضي والتوافق بين استخدامات الأني الأراضي 1-1 أهم المشكلات العمرانية للمدن المصرية نجد في واقعنا المصري أن معظم المدن القائمة حاليًا تعاني كمًا كبيرًا من المشكلات العرانية تتباين في طبيعتها وحجمها تبعًا لاختلاف الظروف البيئية والعمرانية والإجتماعية والإقتصادية

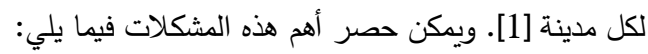

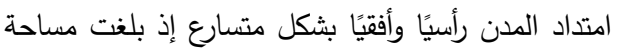
الامتدادات خلال الخمسين سنة الأخيرة أكثر من أربعة الد بلغن أضعاف مسطح المدن قبل ذلك.

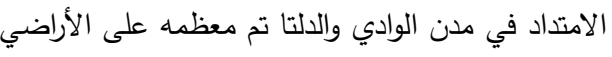
الزراعية الخصبة المحيطة بالمدن مما أدى إلي تآكل

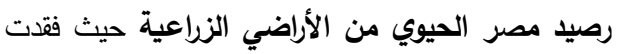

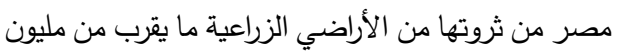

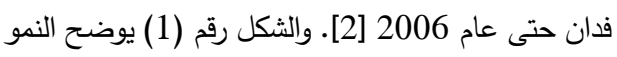
العمراني المستمر على الأراضي الزراعية في مصر وتوقعاته حتى عام 2100.

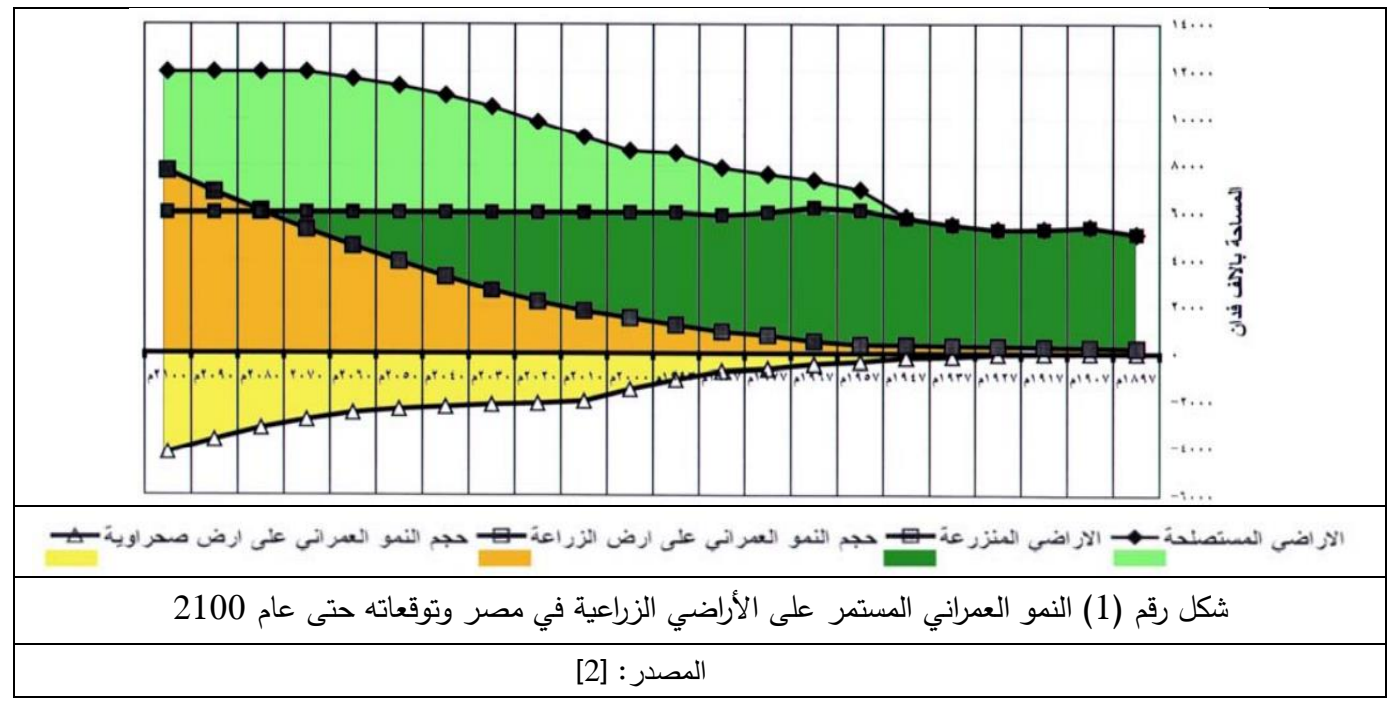


لما سبق فيمكن القول بأن إتباع أسلوب الاستخدام المتعدد للأراضي عند وضع المخططات العمرانية للددن القائمة قد يكون حلًا للتغلب على مشكلة توافر الأراضي الصالحة للتنمية

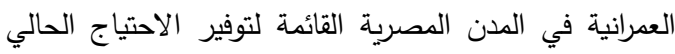
والمستقبلي من الخدمات والإسكان والطرق.

1-3 أدبيات تطبيق الاستخدام المختلط للأراضي

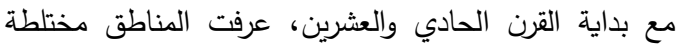

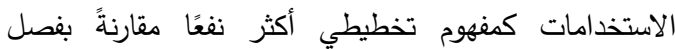
استخدامات الأراضي، وأصبحت استخدامات الأراضي المختلطة

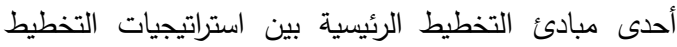

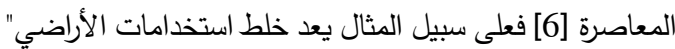

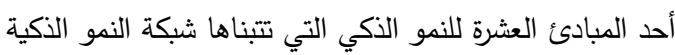
Smart Growth Network the U.S. Environmental وكالة حماية البيئة الأمريكية الجئ Protection Agency الجديدة (Congress for the New Urbanism)، حيث نص أحد مبادئه على أن "تحتوي المجاورات على خليط من

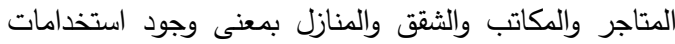

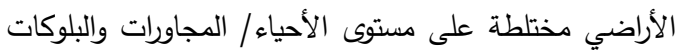

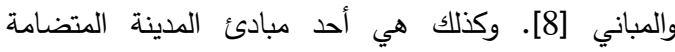
التي تسهم في تحقيق الأستدامة وتقوم على (Compact City)

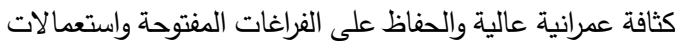
أراضي مختلطة وإعادة إحياء وسط المدينة وأحياء مراكز الأعمال بتوطين استخدامات سكنية واستخدام عالي للمواصلات العامة. وقد فرض موئل الأمم المتحدة UN-Habitat - A New Strategy of Sustainable Neighborhood خمسة مبادئ ثالثها هو خلط استعمالات الأراضي Planning)

وهي [9]:

- - مساحة كافية للشوارع وشبكة شوارع فعالة

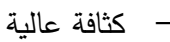

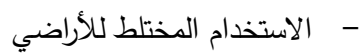

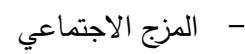

- - مخصيص محدود لاستخدام الأراضي المفرد
- تحديد الأحوزة العمرانية للمدن من خلال مشروع إعداد المخططات الإستراتيجية لهم وذلك لتحديد العجز الحالي والاحتياج المستقبلي من الإسكان والخدمات والأنشطة ومحاولة توفيرهم داخل حدود الحيز العمراني المقترح. - اللجوء إلى تحديد الأولويات في تنفيذ توطين الخدمات بإعطاء الخدمات التعليمية والصحية الأولوية القصوى عند التنفيذ، وإرجاء تتفيذ باقي الخدمات لحين توافر الأراضي اللازمة لها. تخصيص نسبة 25\% من مساحة أراضي التقسيم لتوفير

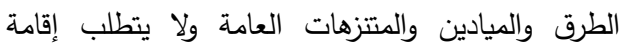
خدمات عامة عليها إذا كانت مساحة الأرض أقل من 5 أفننة، وتخصيص ثلث مساحة أرض التقسيم للمشروعات

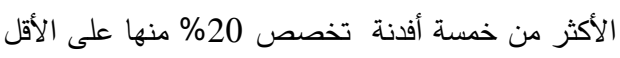
للطرق والباقي يمكن أن يتضمن الخدمات وتتبع إجراءات

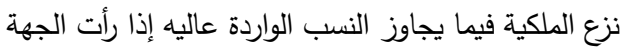

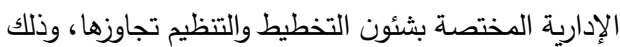
وفقًا لأحكام القانون رقم 10 لسنة 1990 بشأن نزع ملكية

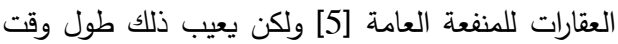
التفاوض على سعر الأرض وتوفير الموارد المالية. - - استخدام سياسة التكثيف الحضري بزيادة كل من الكثافة البنائية والكثافة السكانية ولكن لهذه السياسة مثكلاتها الخاصة مثل التأثير على كفاءة شبكات البنية الأساسية وتوافر وفاعلية الخدمات المجتمعية والتي يتم التعامل معها.

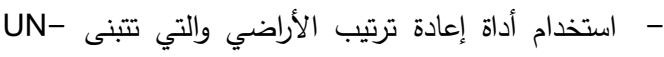

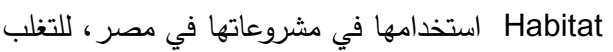
على كل من صغر مسطحات الأراضي الفضاء المملوكة للدولة أو للأفراد والجهات الخاصة، ومشكلة تباعد هذه

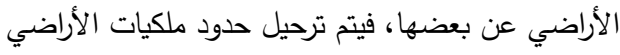

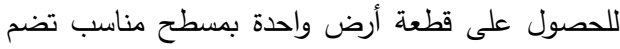
النسبة المخصصة للمنفعة العامة من مجموعة أراض تمت الت لئل اضافتها للحيز العمراني، لكن يقابل استخدام هذه الأداة الكثير من المعوقات، من بينها متطلبات نزع الملكية، وتقدير وتوفير تعويضات المُّلّك. 
وكذلك أقترح Rowley أربعة مستويات لخلط الاستعمالات وهم: مستوى المبنى، مستوى البلوك، مستوى الحي، مستوى المدينة.

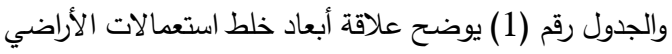

بمستوياتها

جدول رقم (1) علاقة أبعاد خلط استعمالات الأراضي الأربعة بمستوياتها

\begin{tabular}{|c|c|c|c|c|}
\hline الزمني & الرأسي & الأفقي & مشاركة & كالأعاد \\
\hline & & & & المبنى \\
\hline & & & & البلوك \\
\hline & & & & الحي \\
\hline & & & & المدينة \\
\hline
\end{tabular}

المصدر : الباحثة بتصريف عن [11]

ويعترف رولي بأهمية البعد الزمني لأن الاستخدامات

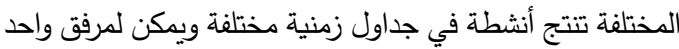
أن يتقاسمه مستخدمون متعددون خلال فترة زمنية معينة، فضلًا عن كونها تتطور مع مرور الوقت مما يمنح المكان طابعًا

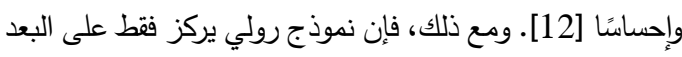

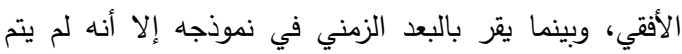

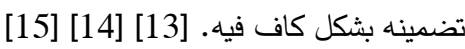

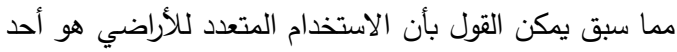

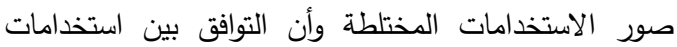
الأراضي المختلفة من العوامل الحاكمة لتطبيقه.

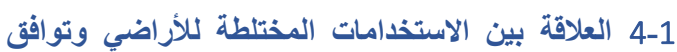
استخدامات الأراضي

بدراسة الأدبيات للاستخدام المختلط للأراضي يتضح أنه يعتبر

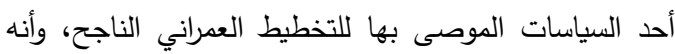

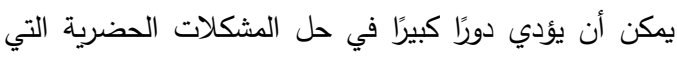

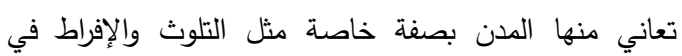

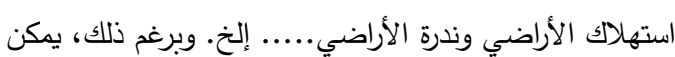

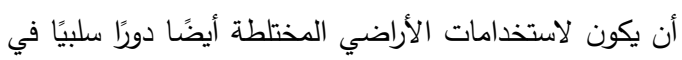
أداء استخدامات الأراضي. ولذا أكدت عدة دراسات على أن الن النيات
وهي تشير إلى مزايا خلط استعمالات الأراضي كجسر لعبور الانقسامات الاجتماعية والإقتصادية في المجتمع، وتعزيز النقل

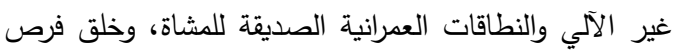

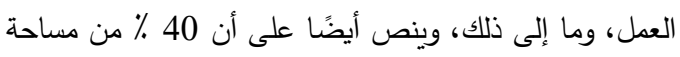
الحي السكني يجب أن تلبي الأنشطة الإقتصادية داخله.

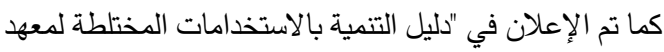

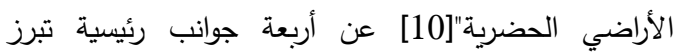
الاستخدام المختلط للأراضي على مستوى قطعة الأرض أو الحي الحي

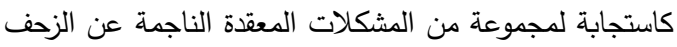
العمراني الذي اجتاح معظم المناطق الحضرية في الولايات

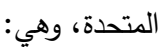

the أ- توفير ثلاثة مؤسسات أو أكثر لتوليد الاقتصاد provision of three or more economy

generating establishments

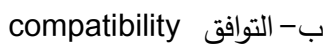
the density of land بكافة استخدامات الأراضي uses

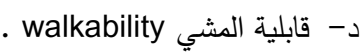
وقد حدد Rowley أبعاد ومستويات خلط استخدامات الأراضي في أربعة أبعاد [11] وهي: - بُعد مشاركة المبنى: ويرتبط بالوظائف المتعددة في المادي

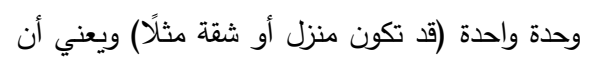
الناس يمكن أن تعمل وتسكن في نفس المبنى. - - البعد الأفقي: وهو تواجد عدد من الاستخدامات بجانب بعضها. - البعد الرأسي : وفيه تتواجد الاستخدامات والوظائف المختلفة بصورة رأسية.

- البعد الزمني : الاستخدامات المتعددة تتم في أوقات

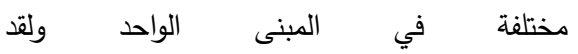

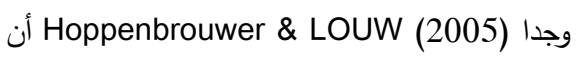
Rowley 
الإضرار بنوعية وجودة البيئة الطبيعية لأي استخدام

يمكن صنعه منها.

إصابة أو تلف الممتلكات أو الحياة النباتية أو الحيوانية

ضرر أو إزعاج مادي لأي شخص

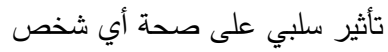

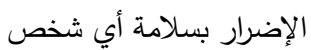

جعل أي ممتلكات أو حياة نباتية أو حيوانية غير لهير

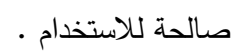

فقدان التمتع بالاستخدام العادي للممتلكات.

التضارب مع السلوك الطبيعي للأعمال.

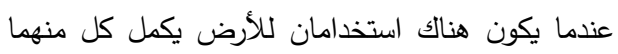

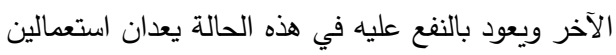
متوافقين وإذا كانا العكس فهما غير متوافقين، أو متنافرين. ويمكن الجزم بأنه تبعًا لظروف الدراسة يختلف نهاج

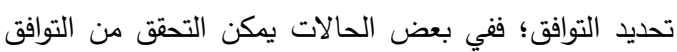

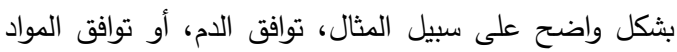

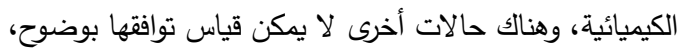

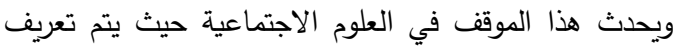

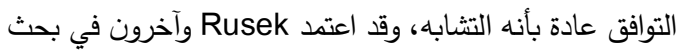
لهم على نفس هذا النهج في مسألة توافق الخدمات واعتبروا أنه

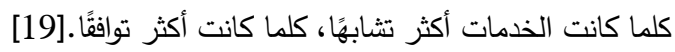

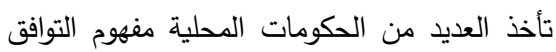

بين فئات استخدام الأراضي في اعتبارها عند تخطيطها

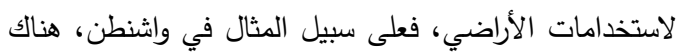

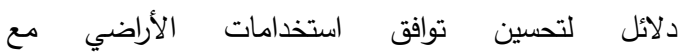
محيطها National Capital Planning Commission

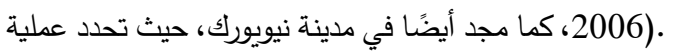

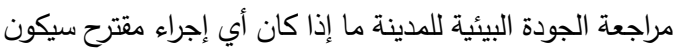

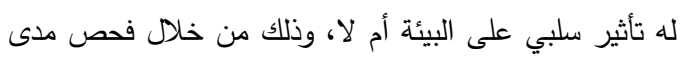

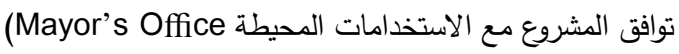

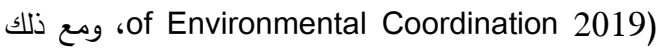
تظل معظم البيانات تتركز على مستوى تصاريح المشروعات

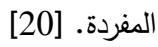

استخدامات الأراضي المختلطة لها علاقة وطيدة بالتوافق بين استخدامات الأراضي، وأوصت بوجوب تضمين تحليل التوافق الأقاض بين استخدامات الأراضي في اعتبارات التخطيط العمراني من ولن فئن

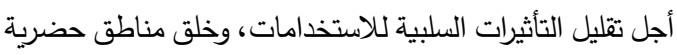
أكثر استدامة. لذا سيتم فيما يلي دراسة التوافق بين استخدامات الأراضي لتوضيح ما هيته وطرق تحديده.

\section{1-5 مفهوم التوافق وأهميته}

التعريف اللغوي للتوافق compatibility كما يوضحه قاموس

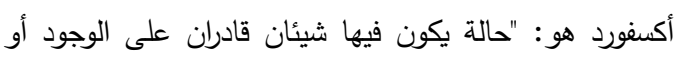

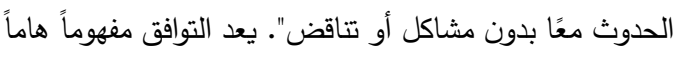

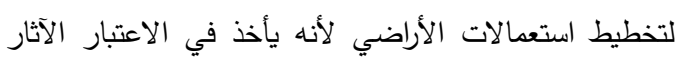

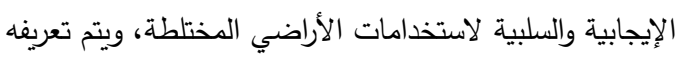

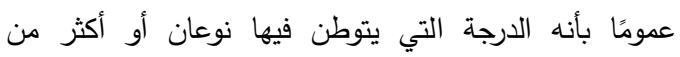

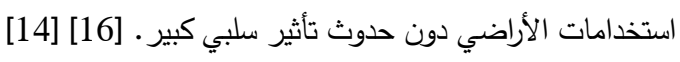

التوافق هو خصائص الاستخدامات أو الأنثطة المختلفة التي

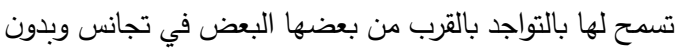

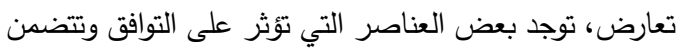

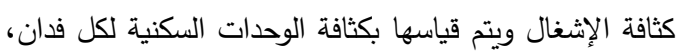

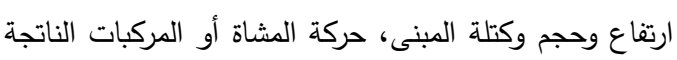

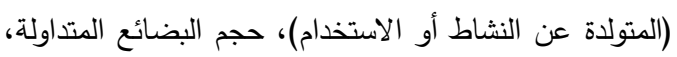

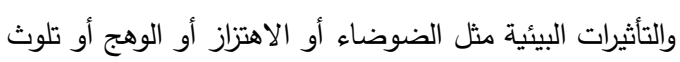

$$
\begin{aligned}
& \text { الهواء أو الإثعاع. [17] } \\
& \text { كما عرف بأنه: }
\end{aligned}
$$

An existing or committed land use or activity " that can co-exist with a neighboring use/activity or uses/activities, without either creating or experiencing one or more off-site 'adverse effect(s)'. [18]

استخدام الأرض أو النشاط الحالي أو المخطط له الذي يمكن

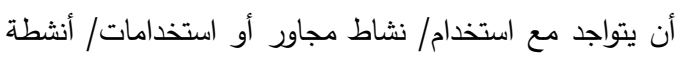

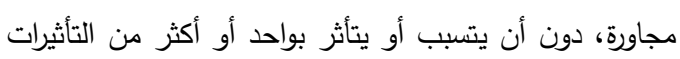

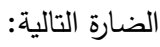


في حالة القرار بتثارك استخدامين نفس قطعة الأرض فيكون

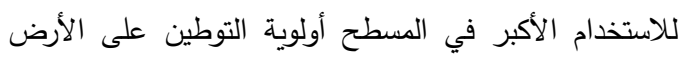
(كاستخدام رئيسي للأرض). 2-الإطار التحليلي

يتم تحليل الدراسات السابقة في محال ت تحديد التوافق بين استخدامات الأراضي للوصول إلى متغيرات تحديد التوافق بين

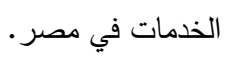

\section{2-1 الدراسات السابقة في مجال تحديد التوافق بين}

$$
\text { استخدامات الأراضي }
$$

توجد العديد من الدراسات لتحديد التوافق بين الخدمات والتي

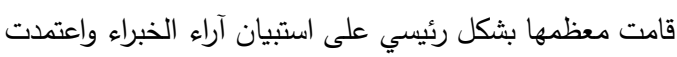

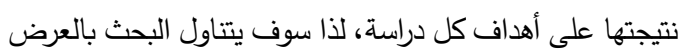

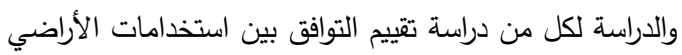

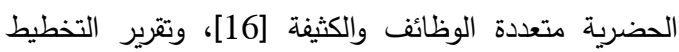

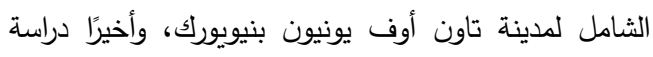
للوقوف على متغيرات تحديد (Vaezi S. et al., 2015) التوافق المستخدمة فيهم ومن ثم الوصول لتحديد متغيرات التوافق لتقيق

المستخدمة بالبحث

أ- التقير التخطيط الثامل لمدينة تاون أوف يونيون بنيويورك

تم وضع عدة اعتبارات لتحديد مدى توافق

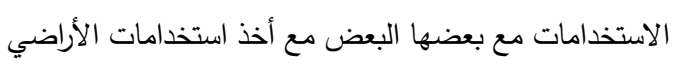

المختلطة في اعتبارها وهي:[23 - الاعتمادية بين استخدامات الأراضي

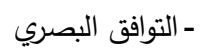

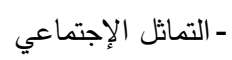
- توليد حركة مرورية -

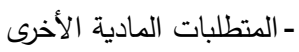

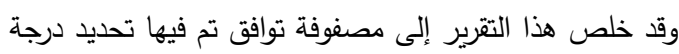
التوافق بين استخدامات الأراضي المختلفة. والجدول (2) يوضح مصفوفة التوافق بين استخدامات الأراضي المختلفة طبقًا

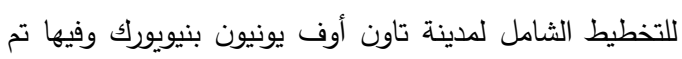
تقسيم التوافق إلى ثلاثة مستويات كالتالي: للتئي - - - استخدامات أراضي متوافقة
تم تقديم بعض الدراسات ذات الصلة بثأن تقييم

تأثيرات توافق استخدام الأراضي من قبل فعلى سبيل المثال Taleai et al. (2007)

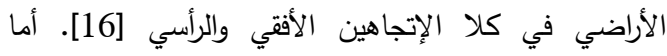

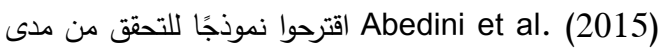

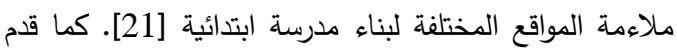

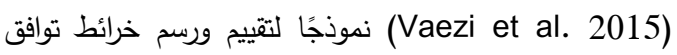
استخدامات الأراضي [22]. وفقًا لـ Taleai et al. (2007) هناك حاجة لبعض الأنشطة على نطاق كلي واسع (macro) ، وبرغم ذلك فقد يكون وجودهم في النطاق الأصغر (Micro) يؤدي إلى توليد تأثيرات خارجية

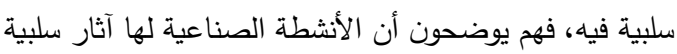
على الأنثطة السكنية على النطاق الصغير (Micro) ولكنها

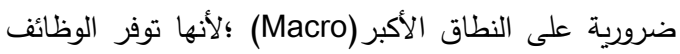

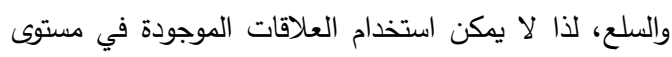

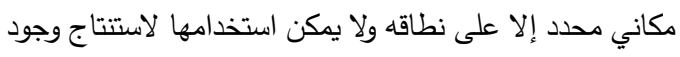

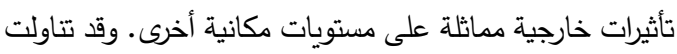

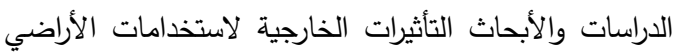

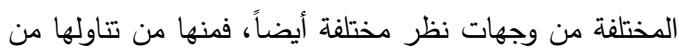
المنظور الإقتصادي أو المنظور البيئي طبقًا لأهداف كل دراسة.

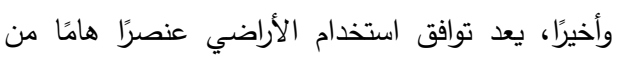

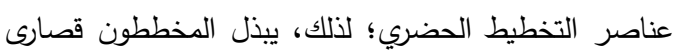
جهدهم لتجنب التعارض بين الوظائف للاستخدامات المتجاورة، لئل

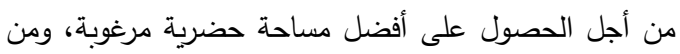

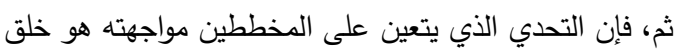

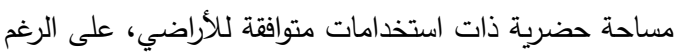

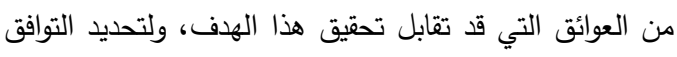

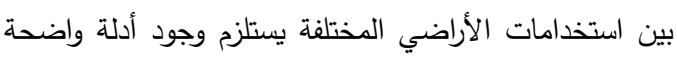

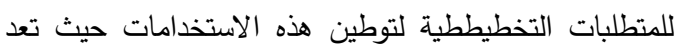

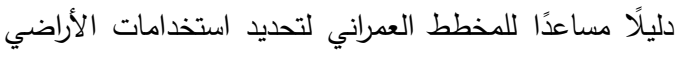

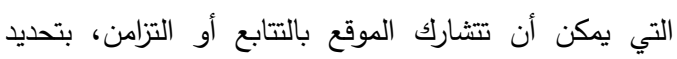

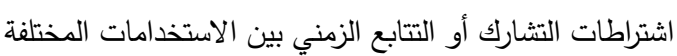

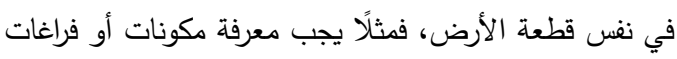

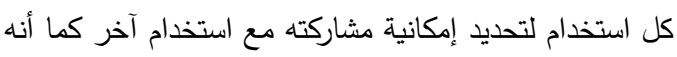


مدى توافقها، تم استخدام طريقة دلفي(Delphi Method) كإطار عمل لبناء مصفوفة التوافق التفصيلية حيث يتغلب هذا

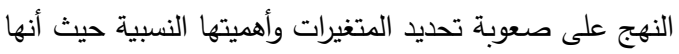
-طريقة دلفي- عملية تكرارية مصممة لتحقيق توافق في الآراء بين مجموعة من الخبراء على موضوع معين، والذي يعد مفيدًا بثكل خاص حينما لا توجد معايير قياسية للتيقييم.

وقد تم تصنيف مستويات التوافق على النحو التالي:[16] • التوافق العالي : استخدامات الأراضي فيها تكون (HC) متجانسة ومتسقة مع بعضها البعض وتتتج درجة عالية من قابليتها للاستخدام usability عندما تكون متجاورة.

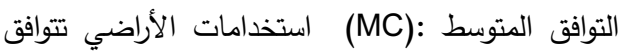
بصورة مقبولة مع بعضها وتتتج مستوى مقبول من قابلية الاستخدام عندما تكون متجاورة.
- -

$$
\text { مع التأثيرات المتولدة عن الاستخدامات) }
$$

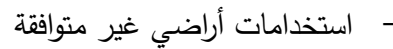

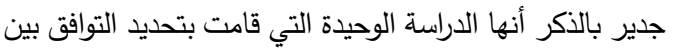
استخدامات الأراضي المختلطة وليس بين استخدامات مفردة.

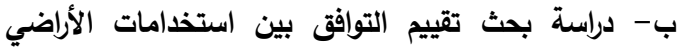

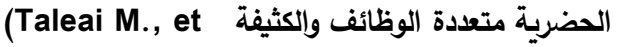

al. ,2006)

Evaluating the compatibility of multi-functional and Intensive urban land uses

- لي هذا البحث تم التوصل لنموذج يصف التوافق العام

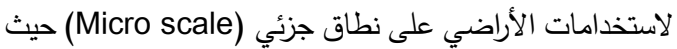
تحدد التعارضات المحتملة بين أنواع استخدام الأراضي المختلفة لإنة جدول (2) مصفوفة التوافق بين الاستعمالات المختلفة طبقا للتخطيط الثامل لمدينة يونيون تاون بنيويورك

- Note: Compatiblity of Mixed Use development is dependent on the proposed mixture of uses.

Source:http://www.townofunion.com 
أولًا: تصنيف أنواع استخدامات الأراضي حسب نشاطها. ثانيًا: مصفوفة توافق استخدامات الأراضي اعتمد تطوير تلك الكي

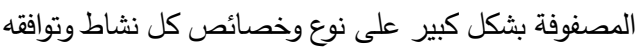

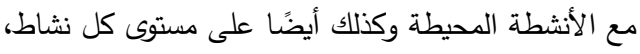

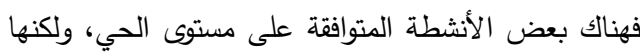

$$
\text { غير متوافقة على مستوى المدينة أو المستوى الإقليمي. }
$$

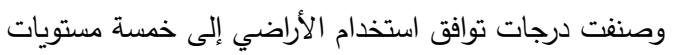

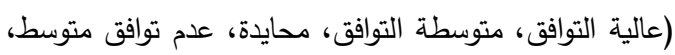
وغير متوافقة للغاية) والجدول (4) يوضح مصفوفة التوافق بين

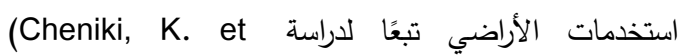
al.,2019) د- عث Rusek, R., et al., 2020) تحت عنوان: Decision support framework for space-use efficiency and arrangement of public services تم تحليل التوافق بين الخدمات بطريقة كمية حيث تتم مقارنة

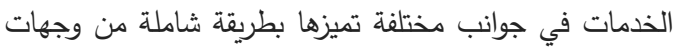
نظر مختلفة وكانت الدراسة تستهدف مشروعات بعينها، وفيها
(MI): عدم التوافق المتوسط / التوافق المنخفض جدًا

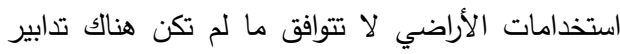

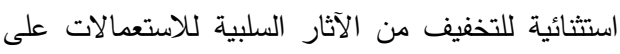
بعضها البعض سوف ينخفض مستوى قابلية الاستخدام usability متجاورة. درجة عالية من عدم التوافق : (HI) يجب أن تكون

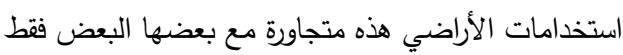

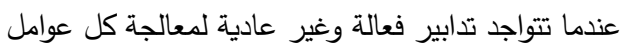
عدم التوافق بفعالية. والجدول(3) يوضح مصفوفة التوافق بين استخدامات الأراضي

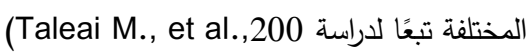
ج- دراسة ( Cheniki, K. et al., 2019 (Tت عنوان: Evaluating Relationship between Mixedland Use and Land-use Compatibility in Algiers Bay تم قياس توافق استخدامات الأراضي باتباع الخطوات التالية: [24] تم اقتراح الخصائص التالية:[25]

جدول (4) مصفوفة التوافق بين استخدمات الأراضي تبعًا لدراسة (Cheniki, K. et al.,2019)

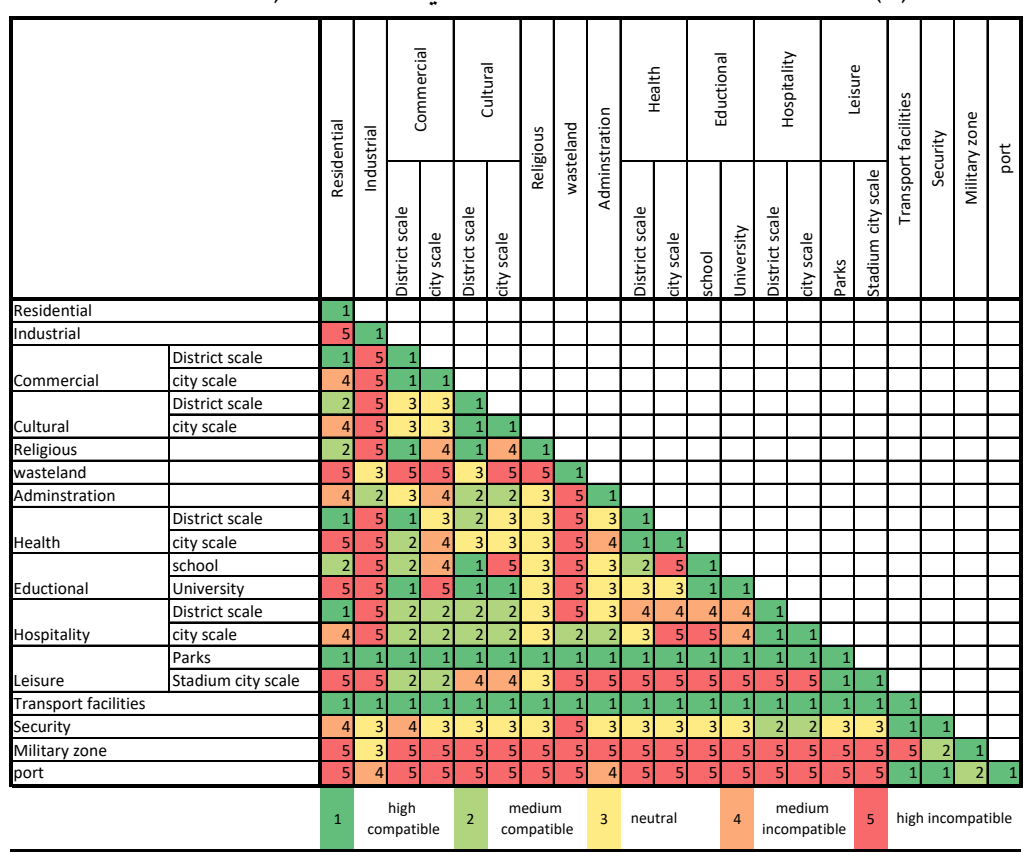

Source: [24] 
جدول (3) مصفوفة التوافق بين استخدامات الأراضي المختلفة تبعًا لدراسة (Taleai M., et al.)

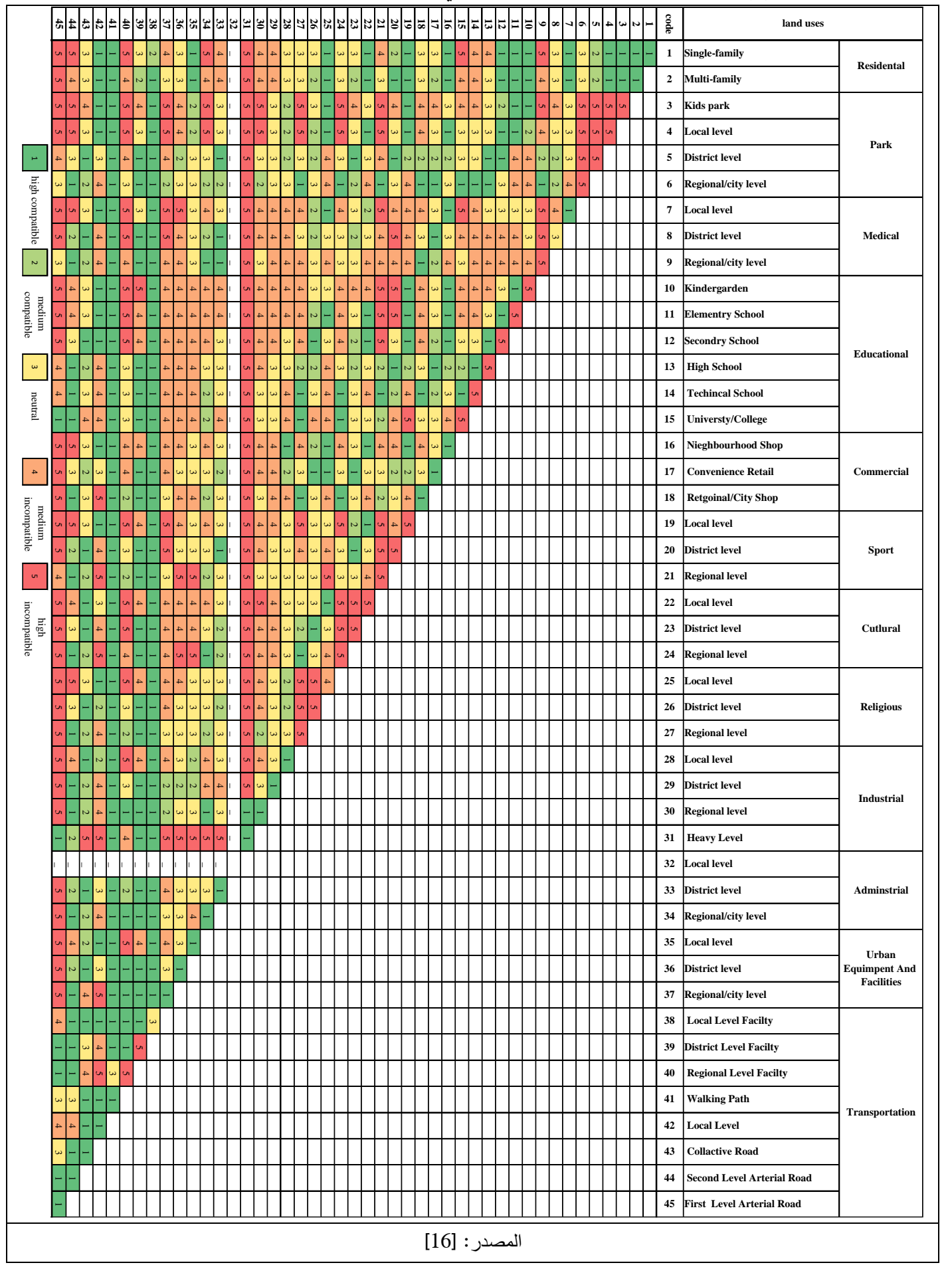


الانتساب - يمثل قسم الإدارة المسؤول عن تقديم

الخدمة، تختلف من مدينة لأخرى بسبب الرخططات

التظيمية المختلفة للمدن.
أولًا: الخصائص التي تصف خدمة من منظور المستخدم :

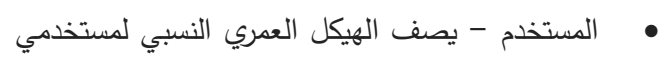

الخدمة: الأطفال والثباب والبالغون وكبار السن.

• الطبيعة - تعكس سمة الخدمة من منظور المستخدم:

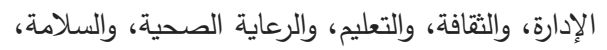

والاجتماعية، والرياضية، والنقل.

الخدمة وغيرهم من المشاركين غير المباشرين).

طريقة تقديم الخدمة - والتي يمكن أن تكون عبر مكتبًا

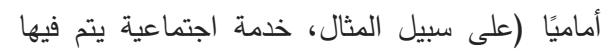

التعامل مع الجمهور)، أو مكتب خلفي (مثل الإدارة).

تم التعبير عن كل من هذه الخصائص كميًا عن طريق تعيين

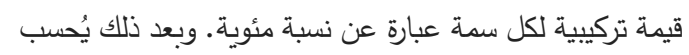

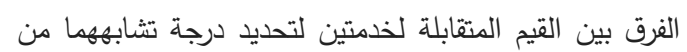

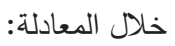

$$
d(S 1, S 2)=100 \%-\left(\frac{1}{2} \sum_{i=1}^{n}|S 1 i-S 2 i|\right)
$$

2-2 متغيرات تحديد التوافق بين استعمالات الأراضي

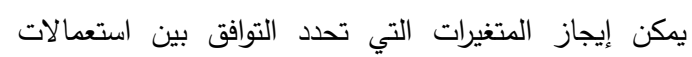
الأراضي طبقا للدراسات السابقة في الجدول (5) التالي:
الحضور (التواجد) - يثير إلى الوضع الذي يتم تقديم

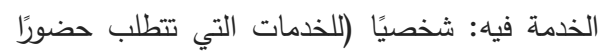

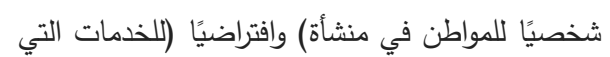
يمكن تقديمها عبر الإنترنت). النطاق - يثير إلى إمكانية الوصول إلى الخدمة. يمكن

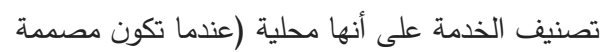

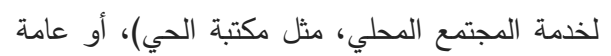

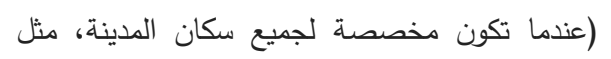
المستشفيات أو الخدمات الإدارية).

ثانيًا الخصائص التي تصف خدمة من منظور الإدارة :

جدول (5) المتغيرات التي تحدد التوافق بين استخدامات الأراضي المختلفة طبقًا للدراسات السابقة

\begin{tabular}{|c|c|c|c|c|}
\hline & & & & \\
\hline 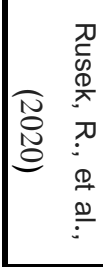 & 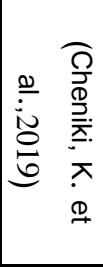 & 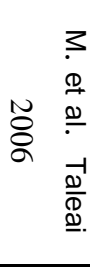 & 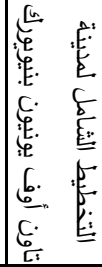 & 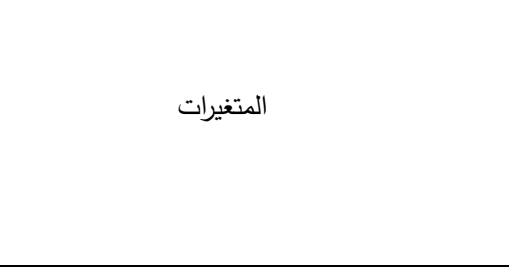 \\
\hline & & & & الاعتمادية بين استخدامات الأراضي \\
\hline & & & & 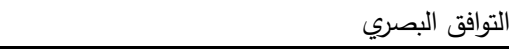 \\
\hline & & & & 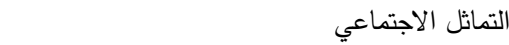 \\
\hline & & & & المتطلبات المادية الاخرى (مسطح - ارتفاع -...) \\
\hline & & & & التأثيرات الخارجية المتولدة من استخدامات الأراضي \\
\hline & & & & المستوى المكاني /النطاق \\
\hline & & & & المستخدم \\
\hline
\end{tabular}




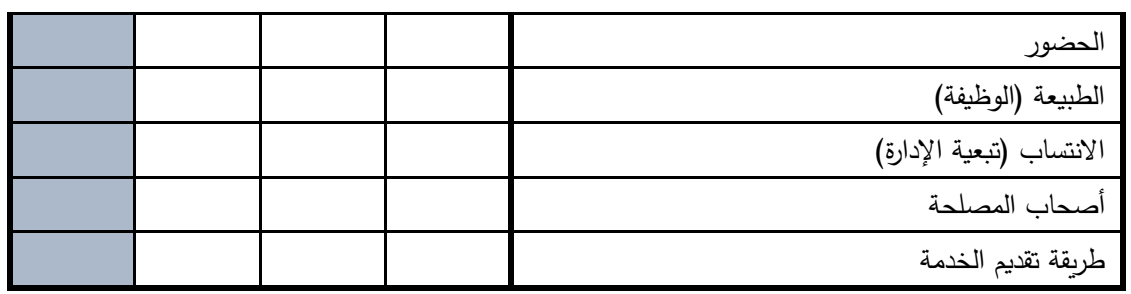

المصدر : الباحثون وفقا للدراسات السابقة لتحديد التوافق بين استخدامات الأراضي

تتطلب تعامل خاص من جهة الأمن والسلامة أو أنها ذات طابع خاص لا يسمح بالتشارك مع استخدامات أخرى وهي ( الخدمات

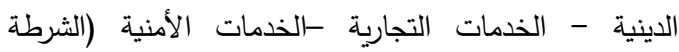
والمطافيء)- الخدمات الصحية)، كذلك تم استبعاد الخدمات

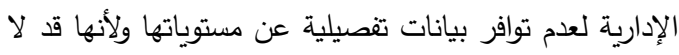

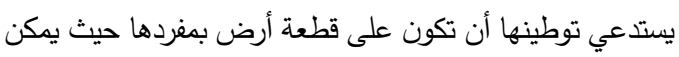
أن تتواجد داخل مبنى وفي أي طابق منه.

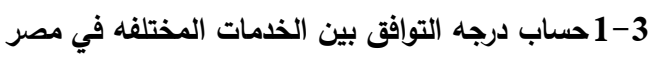

يتم حساب درجه التوافق بين الخدمات المختلفه في مصر بطريقة كمية عن طريق المقارنة بين كل زوج من الخدمات

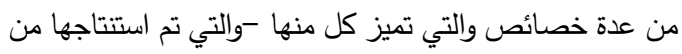

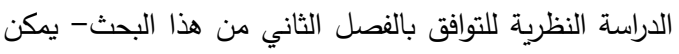

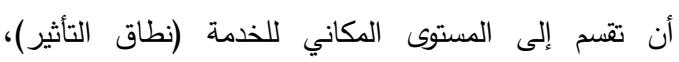
وخصائص المستخدمين، والمتطلبات التخطيطية للخدمة وأخيرًا

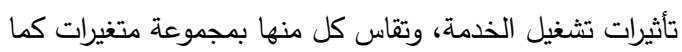
مستوى الخدمة: تتقسم الخدمات تبعا لمستوى أو بالأحرى نطاق تأثيرها إلى : م محلية وهي الخدمات التي تخدم المجاورات والاحياء داخل المدن. o مركزية وهي الخدمات التي تكون على مستوى داء المدن الصغرى المتوسطة. O اقليمية وهي الخدمات التي تكون على مستوى المن المدن الكبرى المليونية وعواصم المحافظات

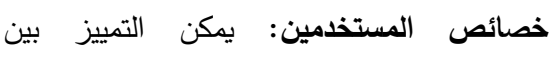
مستخدمي الخدمات ب:
بالنظر في جدول رقم (5) يتضح وجود متغيرات أساسية اتفقت عليها معظم الدراسات لتحديد التوافق بين استخدامات الأراضي

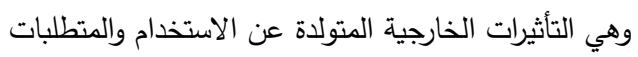
المادية للخدمة ونطاق الخدمة وهي المتغيرات التي سيعتمد

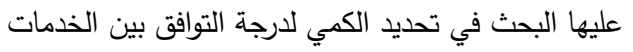

\section{المختلفة.}

وإجمالًا لما سبق فإن مجموعات استخدامات الأراضي في منطقة

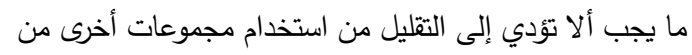

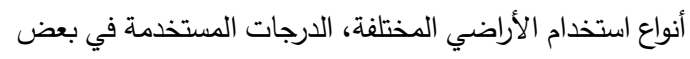

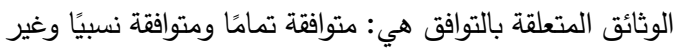

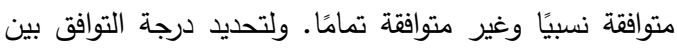

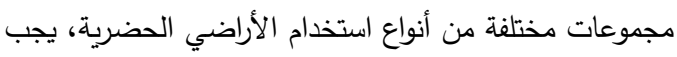

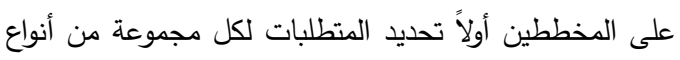

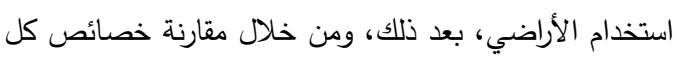

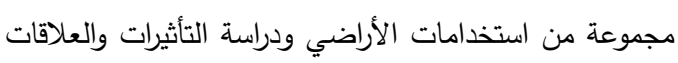
بينها، يمكن للمخطط تحديد درجة التوافق بين أنواع استخدام

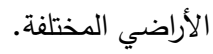

3- مرحلة تحديد درجة التو افق بين الخدمات المختلفة في

مص

أن التوافق بين الاستخدامات المختلفة يعد من العوامل الحاكمة

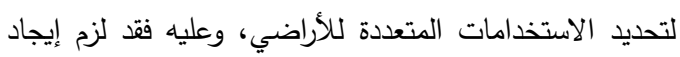
طريقة لحساب درجة التوافق كمئا بين الخدمات حتى تكون أداة

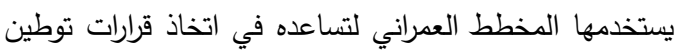
الخدمات بواسطة أسلوب الاستخدام المتعدد للأراضي. وقد تم التمان ترارت توطين

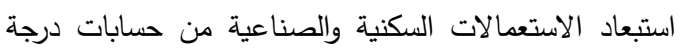

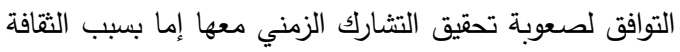

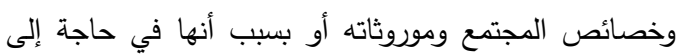
دراسة مستفيضة متخصصة وكذلك استبعدت الاستخدامات التي التي 
الخطورة الناتجة عن تخزين أو استخدام مواد قابلة للاشتعال في محيط الخدمة أو الخطورة الناتجة من الحركة المرورية بسبب التواجد على طريق التيق رئيسي او بالقرب من خطوط السكلك الحديديةأو الاحتياج إلى اتخاذ اجراءات تحد من مصاد الفيادر

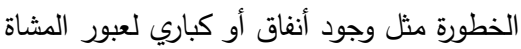

تأثيرات تشغيل الخدمة ويقصد بها التأثيرات المتولدة

$$
\text { عن تشغيل الخدمة وهي تنقسم إلى: تئل }
$$

م توليد حركة مرورية (سواء أكانت حركة مشاة أو الفئ

حركة آلية)

م وتأثيرات بيئية وهي (الضوضاء والمخلفات).

أوقات التشغيل للخدمة وقد تم تقسيمها إلى ثلاث

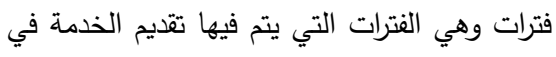

مصر كالتالي:

م صباحاً (من الثامنة صباحاً حتى الرابعة عصرا)

م مساءً ( من الرابعة عصرا وحتى 12 صنباحاً)

م ليلاً (من الثانية عشرة صباحاً وحتى الثامنة

صباحا)

حيث تثكل هذه المتغيرات إطاراً مناسباً لتحديد التثابة

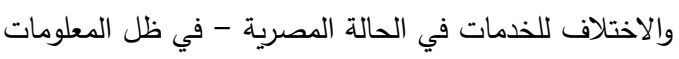
المتاحة -ومن ثم تحديد درجة التوافق بين هذه الخدمات

المختلفة.

يتم التعبير عن كل الخصائص السابقة كمياً عن طريق تحديد

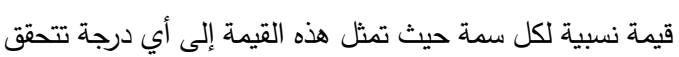
هذه السمة في الخدمة، على سبيل المثال إذا كانت الفئة العمرية

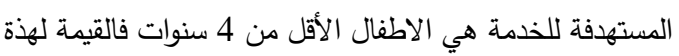
الخدمة تكون 100\% لهذه الفئة وصفر \% لباقي الفئات العمرية، واذا كانت الخدمة تستهدف كل الفئات العمرية فإن كل فئة تأخد

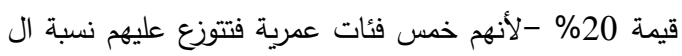

$$
\text { 100\% بهذه الطريقة- وهكذا بما يخص كل متغير . }
$$

يلي ذلك حساب الفرق (بالقيمة المطلقة) بين القيم المتقابلة

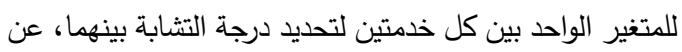
طريق استخدام الصيغة الرياضية التالية [25]:
الفئة العمرية المستهدفة (التي تُقدم اليها الخدمة): وقد قسمت إلى 5 فئات عمريه تتوافق مع مراحل الطفولة والثباب والبالغين وكبار السن وكذلك مع المراحل التعليمية المختلفة في مصر كالتالي:

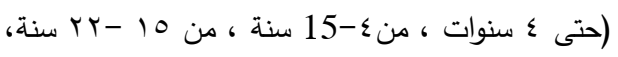

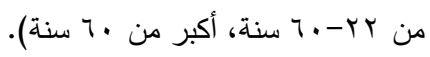

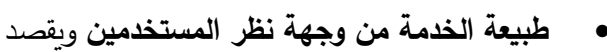
بها تصنيف الخدمة إذا كانت صحية أو تعليمية/ تدريبية أم إدارية -إجتماعية- ثقافية - رياضية أو ترفيهية (7 وظائف) من وجهة نظر المستخدم وليس من جهة تبعيتها الإدارية او تصنيفها الوظيفي. فمثلا

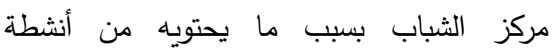
فالمستخدم يستثيد منه في عدة جهات وهي الرياضية (حيث يمكنه ممارسة الرياضة) والترفيهية (بسبب فئن الحفلات والمناسبات التي تقام فيه مثلا) والاجتماعية (لأنه مكان يتم فيه التواصل الاجتماعي بين مرتاديه).أما من الناحية الإدارية فهو يصنف من فن الأن الخدمات الثبابية. المتطلبات التخطيطية للخدمة: وتتقسم إلى أربعة متغيرات كما يلي: الئطي م العلاقة بالطرق ( اذا كانت في حاجة إلى التوطن على طريق فرعي أم طريق رئيسي)

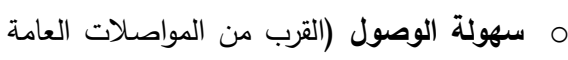
أو وجود مسارات مشاة آمنة) م الحاجة للهدوء : وهو إما ان يكون متطلباً أساسيا لللخدمة فيكون من الضروري عند توطين الخدمة أن تكون بعيدة عن مصادر الضوضاء أو أن يكون متطلباً فرعياً فيسمح عند التوطين بوجود

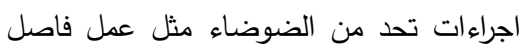
شجري بكثافة معينة أو البناء على أبعاد محددة .

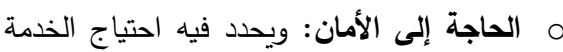
إلى البعد التام عن مصادر الخطورة -والتي يقصد الامند

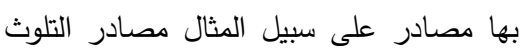

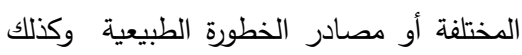


تُحسب درجة التوافق النهائية عن طريق قسمة مجموع

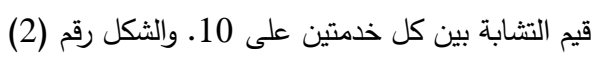

يوضح خطوات حساب درجة التوافق بين الخدمات.

يوضح الجدول (7) قيم متغيرات التوافق للخدمات المختلفة في

مصر حيث تم إعطاء قيم للمتغيرات كنسبة مئوية لكل خدمة

إعتماداً على الآتي:

مستوى الخدمة في هذا المتغير تأخذ الخدمة قيمة

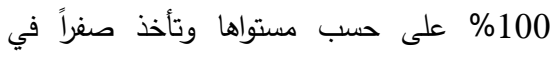
المستويين الآخرين. تم الإعتماد على بيانات تقارير دليل المعدلات

\section{تحديد قيمة نسبية لكل خدمة في كل}

حساب التثابه (برجة التوافق) بين كل خدمتين في

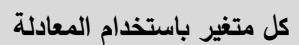
d $(\mathrm{S} 1, \mathrm{~S} 2)=100 \%-\left(1 / 2 \sum_{i=1}^{n}|S 1 i-S 2 i|\right)$

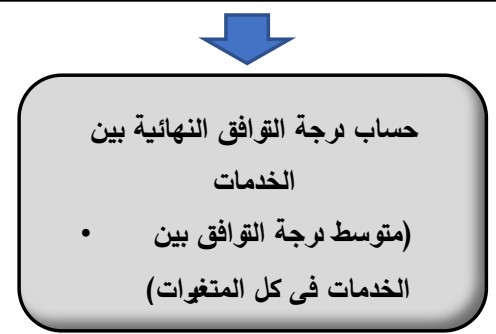

شكل (2) خطوات حساب درجة التوافق بين الخدمات

المصدر : الباحثون

والمعايير التخطيطية للخدمات بجمهورية مصر العربية من إعداد

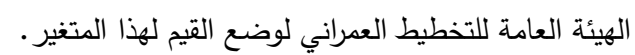

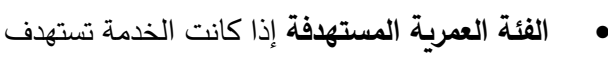

فئة واحدة فتكون النسبة فيها 100\% وباقي الفئات

صفر واذا كانتت تخدم أكتر من فئة فتقسم النسبة

بينهم بالتساوي بمعنى لو أن الخدمة تستهدف 4

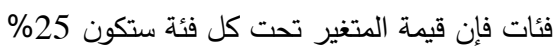

$$
d(S 1, S 2)=100 \%-\left(\frac{1}{2} \sum_{i=1}^{n}|S 1 i-S 2 i|\right)
$$

حيث (S1) تمثل قيم متغيرات الخدمة الأولى و(S2) قيم متغيرات الخدمة الثانية

وللحصول على قيمة النسبة المئوية التي تعكس درجة التثابه

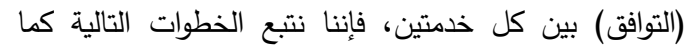

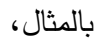

بفرض أن هناك (خدمتين 1و2) يتم حساب درجة التثابة (التوافق بينهما) عبر متغير الفئة المستهدفة الموضح باكن (الجدول رقم (6) من خلال إتباع الخطوات التالية: جدول (6) قيم متغير الفئة المستهدفة للخدمتين

\begin{tabular}{|c|c|c|c|c|c|}
\hline من 60 سنة & $\begin{array}{c}\text { من } 22 \\
\text { 60سنة }\end{array}$ & $\begin{array}{c}\text { من } \\
\text { 22 }\end{array}$ & سن 45 - 15 & سنو 4 سنوات & المستئة \\
\hline 0 & 0 & 0 & 0 & 100 & خدمة 1 (\%) \\
\hline 20 & 20 & 20 & 20 & 20 & $\begin{array}{rr} & \\
& \\
& \end{array}$ \\
\hline
\end{tabular}

1و2الافتراضيتين

المصدر : الباحثون

• يتم التعويض في المعادلة السابقة:

$d(S 1, S 2)=100 \%-\left(\frac{1}{2}(|100-20|+\mid 0-\right.$ $20|+| 0-20|+| 0-20|+| 0-20 \mid))$ $=100 \%-\left(\frac{1}{2}(80+20+20+\right.$ $20+20))$

$$
=100 \%-\left(\frac{1}{2}(160)\right)
$$

$$
=20 \%
$$

وعليه تكون درجة التشابه بين الخدمتين 1و2 بالنسبة لمتغير

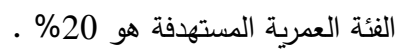

• تطبيق المعادلة على كل قيم المتغيرات العشرة لقياس التشابه الاجمالي بين الخدمات. 
صفر ، كذلك وقد تم الإعتماد على بيانات تقارير دليل المعدلات والمعايير التخطيطية للخدمات بجمهورية مصر العربية من إعداد الهيئة العامة للتخطيط العمراني ومتطلبات استعمالات الأراضي Urban land use planning المختلفة من كتاب [26] لوضع القيم لهذا المتغير. الحاجة إلى الأمان: تم وضع قيم المتغيرات واللجوء إلى نفس المراجع مثلما سبق شرحه في المتغير السابق. توليد حركة مرورية: قد أُتبعت نفس الطريقة ونفس

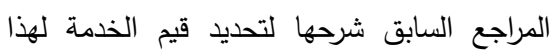
المتغير التأثيرات البيئية : قد أتُبعت نفس الطريقة السابق شرحها واعتُمد على نشرات وتقارير الجهاز المركزي للتعبئة العامة والإحصاء لحالة البيئة في مصر لتحديد قيم الخدمة لهذا المتغير . الثكل (3) يوضح طريقة حساب درجة التوافق بين الخدمات في مصر كما يوضح الجدول رقم (8) مصفوفة درجة التوافق النهائية بين الخدمات المختلفة في مصر
والفئة الخامسة صفر • وقد تم الإعتماد على بيانات تقارير دليل المعدلات والمعايير التخطيطية للخدمات بجمهورية مصر العربية من إعداد الهيئة العامة للتخطيط العمراني لوضع القيم لهذا المتغير.

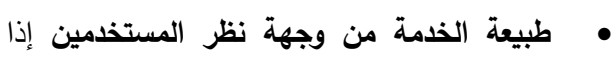
كانت الخدمة تؤدي وظيفة واحدة للمستخدم فتأخذ قيمة 100\% فيها وتأخذ باقي الوظائف صفر • وإذا كانت الخدمة تؤدي للمستخدم وظيفتين فإنها تأخذ 50 في كل منهما وصفراً في البقية وإذا كانت تؤدي أكثر من وظيفة يتم تقسيم ال100\% على عدد الوظائف والبقية تأخذ القيمة صفر . العلاقة بالطرق تضم متغيرين إذا كانت الخدمة تتطلب وجود أحدهما فقط فتأخذ القيمة 100\% وفي لـئ الآخر صفر وقد تم الإعتماد على بيانات تقارير دليل المعدلات والمعايير التخطيطية للخدمات بجمهورية مصر العربية من إعداد الهيئة العامة للتخطيط العمراني لوضع القيم لهذا المتغير ·

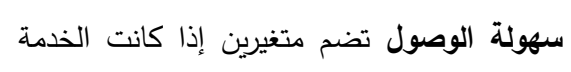
تتطلب وجود أحدها فقط فتأخذ فيه القيمة 100\% وفي الآخر صفر واذا كانت تتطلب وجود المتغيرين اخذت 50\% في كل منهما، كذلك وقد تم الإعتماد على بيانات تقارير دليل المعدلات والمعايير التخطيطية للخدمات بجمهورية مصر العربية من إعداد الهيئة العامة للتخطيط العمراني لوضع القيم

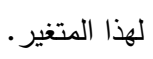
الحاجة للهدوء: تضم متغيرين فإذا كانت الخدمة تتطلب وجود أحدهما فقط فتأخذ فيه القيمة 100\% وفي الآخر صفر واذا كانت تحتمل أن تتطلب وجود المتغيرين اخذت 50\% في كل منهما، وإذا كانت الخدمة لا تتأثر بهذا المتغير تكون قيم النسب فيها 


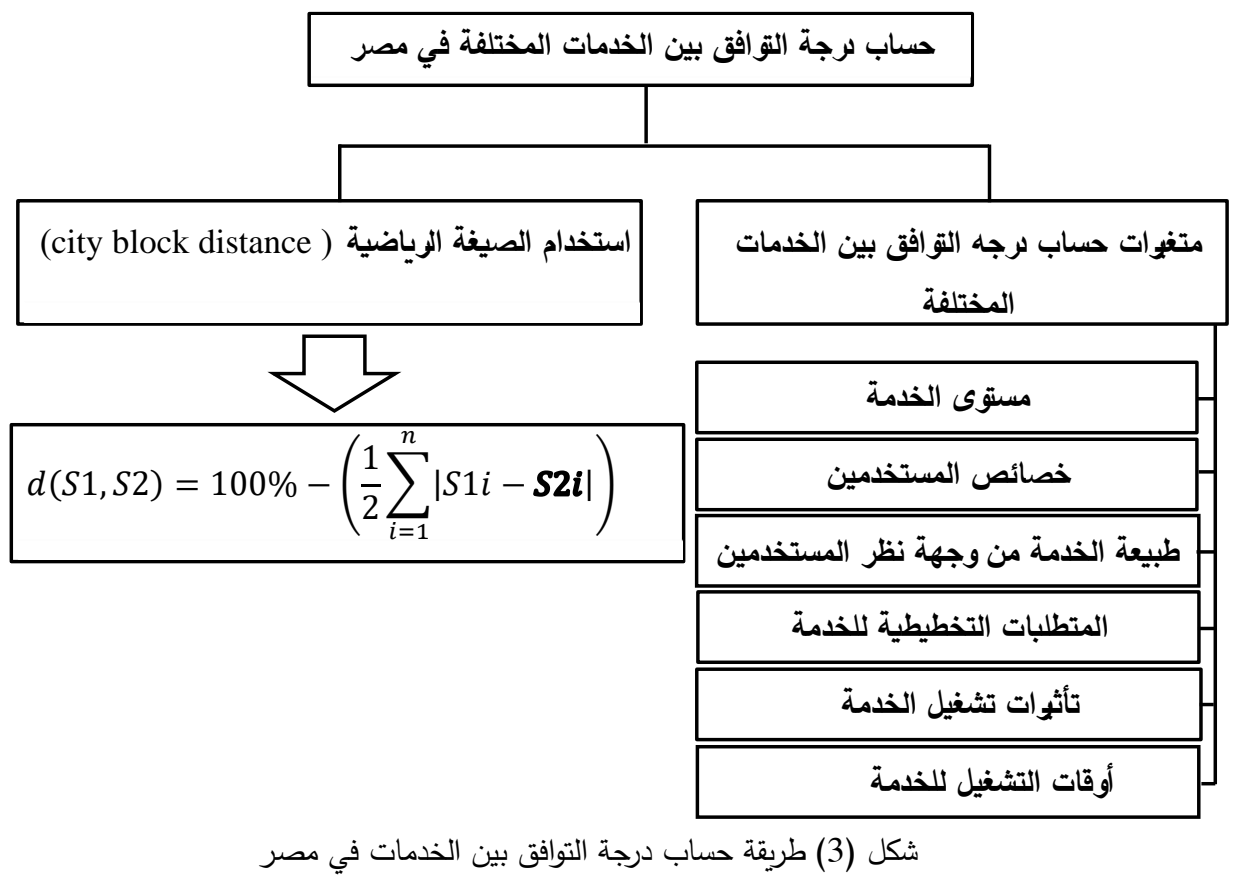

من صفر). حيث توفر قيمة درجة التوافق قاعدة للمقارنة بين

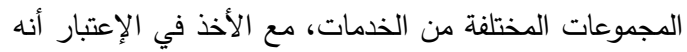

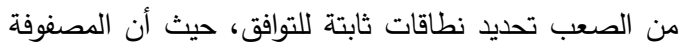
تم تقسيم درجة التوافق حسب قيمتها كما تظهر في جدول رقم

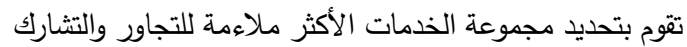
في الموقع. فعلى سبيل المثال، إذا كانت درجة التوافق بين

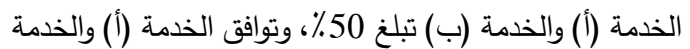
(ج) تبلغ 70\%، فهذا معناه أنه يوصى بالجمع بين الخدمات

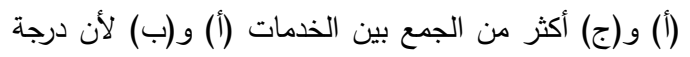

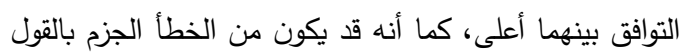

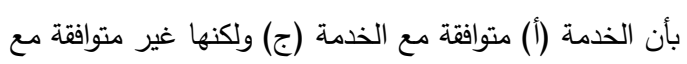

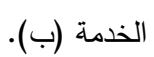

$$
\begin{aligned}
& \text { - - - - - } \\
& \text { - - - - } \\
& \text { - - - - - - }
\end{aligned}
$$

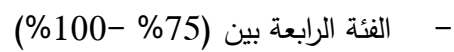

تحدد مصفوفة التوافق مجموعات الخدمات المتوافقة والتي يمكن توطنيها بالقرب من بعضها (قيم قريبة من 100) ومجموعات الخدمات التي يجب تجنب توطنيها بالقرب من بعضها (قيم قريبة 
جدول (7) يوضح قيم (نسب) متغيرات التوافق للخدمات المختلفة في مصر

\begin{tabular}{|c|c|c|c|c|c|c|c|c|c|c|c|c|c|c|c|c|c|c|c|c|c|c|c|c|c|c|c|c|}
\hline & & & & & & & & & & & (1) & & & & & 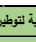 & 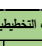 & النطبات & & & & لوتكنين & 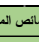 & & & & & \\
\hline & & & & & & & & كقات التئة & & لي بري بيية & & the & & 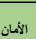 & & ; & & 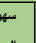 & & & & & & & & له هي الظمة & & \\
\hline & & ? & 雃: & & & है & & 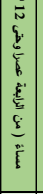 & & 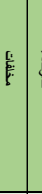 & & 1 & & & 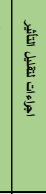 & & 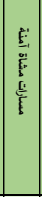 & & & & 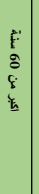 & & & & & $=$ & ' $\xi$ & \\
\hline 0 & 0 & 0 & \begin{tabular}{l|l} 
\\
\end{tabular} & \begin{tabular}{|l|l|}
0 \\
\end{tabular} & 100 & 0 & 0 & 0 & 100 & 50 & & 10 & & 10 & 0 & 100 & 100 & 0 & 0 & 100 & 0 & 0 & & 100 & 00 & \begin{tabular}{l|l|}
0 & 0 \\
\end{tabular} & 100 & |سرسة تلثير أناسي \\
\hline & 0 & 0 & \begin{tabular}{|l|l|l|l} 
\\
\end{tabular} & \begin{tabular}{|l|l|l|l|l} 
& \\
\end{tabular} & 100 & 0 & & & 100 & 505 & & 0 & 5 & 50 & 50 & 50 & 50 & & 100 & 0 & & & & & & & & \\
\hline 0 & 0 & 0 & 0 & \begin{tabular}{|l|l|}
0 & \\
\end{tabular} & 100 & 0 & 0 & 0 & 100 & 505 & & 0 & 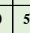 & 50 & \begin{tabular}{|l|}
50 \\
\end{tabular} & 50 & 50 & 50 & 100 & 0 & 0 & & 00 & 0 & & \begin{tabular}{ll|l}
00 & 0
\end{tabular} & 0 & إمدرسة تليه فن \\
\hline 0 & 0 & 0 & 0 & 0 & 100 & 0 & 0 & 50 & 50 & 505 & & \begin{tabular}{l|l}
0 & 5 \\
\end{tabular} & 56 & 50 & 50 & 50 & 50 & 50 & 100 & 0 & 0 & & & 0 & \begin{tabular}{l|l}
0 & 10 \\
\end{tabular} & $\begin{array}{lll}00 & 0 \\
\end{array}$ & 0 & باسية \\
\hline & 0 & 0 & \begin{tabular}{l|l}
0 \\
\end{tabular} & 100 & & 0 & 0 & 0 & 100 & 0 & & \begin{tabular}{l|l}
01 \\
0
\end{tabular} & & 10 & 0 & 100 & 100 & 0 & & 100 & 25 & 25 & & 25 & & & & 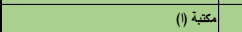 \\
\hline 0 & 0 & 0 & 0 & 100 & 0 & 0 & 0 & 0 & 100 & 0 & & \begin{tabular}{l|l}
0 & 5 \\
\end{tabular} & 5 & 50 & \begin{tabular}{|l|}
50 \\
\end{tabular} & 50 & 50 & 50 & 100 & 0 & 25 & 25 & 25 & 25 & 0 & \begin{tabular}{l|l}
0 & 100 \\
\end{tabular} & 0 & 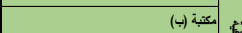 \\
\hline & 33 & 0 & 33 & 34 & & 0 & 0 & 50 & 50 & 50 & & \begin{tabular}{l|l}
05 & 5 \\
\end{tabular} & s & 50 & 50 & 50 & 50 & 50 & 100 & 0 & 25 & 25 & & 25 & & \begin{tabular}{l|l}
0 & 100 \\
\end{tabular} & 0 & بيت ثلافة \\
\hline & 33 & 0 & 33 & 34 & & 0 & 0 & 50 & 50 & 50 & & \begin{tabular}{l|l}
0 & 5 \\
\end{tabular} & 5 & 50 & 50 & 50 & 50 & 50 & 100 & 0 & 25 & 25 & 25 & 25 & & $\begin{array}{ll}00 & 0\end{array}$ & 0 & لتهر تافة \\
\hline & 33 & 34 & 33 & 0 & 0 & 0 & 0 & 50 & 50 & 01 & & 10 & 0 & 10 & 0 & $\begin{array}{ll} \\
\end{array}$ & 100 & 0 & 0 & 100 & 25 & 25 & 25 & 25 & $\begin{array}{ll}0 & 0 \\
\end{array}$ & \begin{tabular}{l|l|}
0 & 0 \\
\end{tabular} & 100 & ملياعب بمتودة (ع) \\
\hline & 33 & 34 & 33 & \begin{tabular}{|l}
0 \\
\end{tabular} & & 0 & 0 & 50 & 50 & & & \begin{tabular}{l|l}
0 & 5 \\
\end{tabular} & 0 & 10 & 0 & 0 & 100 & 0 & 0 & 100 & 25 & 25 & & & & & 100 & ملاعب بشتردة (ب) \\
\hline & 33 & 34 & 33 & 0 & & 0 & 0 & 50 & 50 & 01 & & \begin{tabular}{l|l}
0 & 5 \\
\end{tabular} & 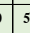 & 50 & 0 & 0 & 50 & 50 & 100 & 0 & 25 & 25 & 25 & 25 & & \begin{tabular}{l|l|}
0 & 0 \\
\end{tabular} & 100 & |نادي بابض (ع) \\
\hline & 33 & 34 & 33 & 0 & & 0 & 0 & 50 & 50 & 505 & & \begin{tabular}{l|l}
0 & 5 \\
\end{tabular} & 56 & 50 & 0 & 0 & 50 & 50 & 100 & 0 & 25 & 25 & 25 & 25 & 0 & \begin{tabular}{l|l}
0 & 100 \\
\end{tabular} & & ملاعب بنتوة (i) (1) \\
\hline & 33 & 34 & 33 & 0 & & 0 & 0 & 50 & 50 & 505 & & $\begin{array}{l}05 \\
0\end{array}$ & & 50 & 0 & 0 & 50 & 50 & 100 & 0 & 25 & 25 & & 25 & & \begin{tabular}{l|l}
0 & 100 \\
\end{tabular} & 0 & |نادي برباضي (ب) \\
\hline & 33 & 34 & 33 & 0 & 0 & 0 & 0 & 50 & 50 & 5 & & \begin{tabular}{l|l}
0 & 5 \\
\end{tabular} & 5 & 50 & 0 & 0 & 50 & 50 & 100 & 0 & 25 & 25 & 25 & 25 & \begin{tabular}{l|l}
0 & 10 \\
\end{tabular} & $\begin{array}{lll}00 & 0\end{array}$ & 0 & \\
\hline & 33 & 34 & 33 & 0 & & 0 & 0 & 50 & 50 & 505 & & \begin{tabular}{l|l}
0 & 5 \\
\end{tabular} & 56 & 50 & 0 & 0 & 50 & 50 & 100 & 0 & 20 & 20 & & 202 & & $\begin{array}{lll}00 & 0 \\
\end{array}$ & 0 & | بتاد (ب) \\
\hline & 0 & 50 & \begin{tabular}{|l|l|}
0 \\
\end{tabular} & 0 & 50 & 0 & 33 & 33 & 34 & 01 & & \begin{tabular}{l|l}
0 & 5 \\
\end{tabular} & 56 & 50 & 0 & 0 & 50 & & 100 & 0 & 25 & 25 & 25 & 25 & \begin{tabular}{l|l}
0 & 10 \\
\end{tabular} & \begin{tabular}{c|c|}
00 & 0
\end{tabular} & 0 & 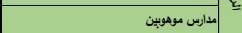 \\
\hline & 33 & 34 & 33 & 0 & 0 & 0 & 0 & 50 & 50 & $50 \mathrm{~s}$ & & 05 & ${ }^{5} t$ & 50 & 0 & 0 & 50 & 50 & 100 & 0 & 20 & 20 & 20 & 202 & 10 & $\begin{array}{ll}00 & 0\end{array}$ & 0 & (1) \\
\hline & 50 & 0 & 50 & \begin{tabular}{|l|l|} 
& \\
\end{tabular} & & 0 & 33 & 33 & 34 & 100 & & \begin{tabular}{l|l}
0 & 5 \\
\end{tabular} & 5 & 50 & 0 & \begin{tabular}{|l|l} 
& 0 \\
\end{tabular} & 50 & 50 & 100 & 0 & 25 & 25 & & $25 \mathrm{C}$ & & $\begin{array}{lll}00 & 0\end{array}$ & & |شنق الرباضيني \\
\hline & 0 & 50 & \begin{tabular}{l|l|l|} 
& \\
\end{tabular} & 0 & & 0 & 33 & \begin{tabular}{l|l}
33 \\
\end{tabular} & 34 & 100 & & \begin{tabular}{l|l}
0 & 5 \\
\end{tabular} & 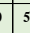 & 50 & 50 & 50 & 50 & 50 & 0 & 100 & 25 & 25 & 25 & 25 & $\begin{array}{l}0.10 \\
0\end{array}$ & $\begin{array}{lll}00 & 0 \\
00\end{array}$ & 0 & ودة طب برباضي \\
\hline & 33 & 34 & 33 & \begin{tabular}{|l|l|}
0 \\
\end{tabular} & 0 & 0 & 0 & 50 & 50 & 50 & & \begin{tabular}{l|l}
0 & 5 \\
\end{tabular} & 56 & 50 & 0 & 0 & 50 & 50 & 100 & 0 & 25 & 25 & 25 & 25 & $\begin{array}{ll}0 & 10 \\
0\end{array}$ & $\begin{array}{lll}00 & 0\end{array}$ & 0 & مشرئ تسية بياضية \\
\hline & 33 & 34 & 33 & \begin{tabular}{|l}
0 \\
\end{tabular} & & 0 & 33 & 33 & 34 & $50 \mathrm{~s}$ & & \begin{tabular}{l|l}
0 & 5 \\
\end{tabular} & 5 & 50 & 0 & \begin{tabular}{|l}
0 \\
\end{tabular} & 50 & 50 & 100 & 0 & 25 & 25 & & 25 & & \begin{tabular}{ll|}
00 & 0 \\
\end{tabular} & 0 & قربرة أريبية: \\
\hline & 50 & 0 & \begin{tabular}{l|l|l|} 
& \\
\end{tabular} & 50 & & 0 & 0 & 0 & 100 & 0 & & \begin{tabular}{l|l}
0 & 10 \\
\end{tabular} & \begin{tabular}{l|l}
0 & 0 \\
\end{tabular} & 10 & 0 & 100 & 100 & 0 & 0 & 100 & 0 & 0 & & $100 \mathrm{C}$ & \begin{tabular}{l|l}
0 & 0 \\
\end{tabular} & \begin{tabular}{l|l|}
0 & 0 \\
\end{tabular} & 100 & مكتبة طلا \\
\hline & 50 & 0 & 0 & 0 & 50 & 0 & 0 & 0 & 100 & 50 & & \begin{tabular}{l|l}
10 \\
10
\end{tabular} & 0 & 10 & 0 & 100 & 100 & 0 & 0 & 100 & 0 & 0 & & \begin{tabular}{l|l}
0 & 16 \\
\end{tabular} & \begin{tabular}{l|l}
00 & 0 \\
\end{tabular} & \begin{tabular}{l|l}
0 & 0 \\
\end{tabular} & & كادر ضشاتة \\
\hline & 34 & 0 & 0 & 33 & & 0 & 0 & 50 & 50 & 50 & & 10 & & 10 & \begin{tabular}{l|l|}
0 & 0 \\
\end{tabular} & 100 & 100 & 0 & 0 & 100 & 0 & 0 & & 100 & 0 & \begin{tabular}{l|l}
0 & 0 \\
\end{tabular} & 100 & |نادي لطل \\
\hline & 34 & 0 & 33 & \begin{tabular}{|l|l|}
33 & \\
\end{tabular} & & 0 & 0 & 50 & 50 & 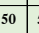 & & 0 & 0 & 10 & \begin{tabular}{l|l|}
0 & 0
\end{tabular} & 100 & 100 & 0 & 0 & 100 & 20 & 20 & & 202 & 20 & \begin{tabular}{|l|l|}
0 & 0 \\
\end{tabular} & 10 & بدية طلك \\
\hline & 34 & 0 & 33 & 33 & & 0 & 0 & 50 & 50 & 50 & 0 & 0 & $5 t$ & 50 & 50 & 50 & 50 & 50 & 0 & 100 & 33 & 34 & 33 & 0 & 0 & \begin{tabular}{l|l|}
0 & 0 \\
\end{tabular} & & كانيا نساتي \\
\hline & 50 & 0 & 0 & 0 & & 50 & 0 & 0 & 100 & 50 & & 0 & 16 & 0 & 50 & 50 & 50 & 50 & 0 & 100 & 20 & 20 & 20 & 202 & 0 & \begin{tabular}{|l|l|}
0 & 0 \\
\end{tabular} & 100 & 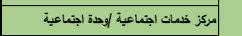 \\
\hline & 50 & 0 & 0 & 50 & & 0 & 0 & 50 & 50 & 50 & & \begin{tabular}{l|l}
0 & 5 \\
\end{tabular} & 10 & \begin{tabular}{l|l}
0 & 0 \\
\end{tabular} & 50 & 50 & 50 & 50 & 0 & 100 & 0 & 50 & 50 & 0 & 0 & \begin{tabular}{l|l|}
0 & 0 \\
\end{tabular} & & |نابي الشاعي ثافي \\
\hline & 50 & 0 & 0 & 50 & & 0 & 0 & 50 & 50 & 100 & & \begin{tabular}{l|l}
0 & 5 \\
\end{tabular} & 10 & $\begin{array}{ll}0 & 0 \\
0\end{array}$ & 50 & 50 & 50 & 50 & 0 & 100 & 0 & 50 & ${ }_{50}$ & 0 & & \begin{tabular}{l|l|}
0 & 0 \\
\end{tabular} & 100 & لنابي فلاع اجشاعي \\
\hline & 50 & 0 & 0 & 50 & & & 0 & 50 & & 100 & & & & 10 & 0 & 100 & 50 & 50 & 0 & 100 & 100 & 0 & & 0 & 0 & \begin{tabular}{l|l}
0 & 100 \\
\end{tabular} & 0 & الثائي معائكيار السن \\
\hline & 33 & 0 & 0 & 0 & 34 & 33 & 0 & 50 & 50 & $50 \mathrm{~s}$ & & 0 & 56 & 50 & 50 & 50 & 50 & 50 & 100 & 0 & 25 & 25 & 25 & 25 & 0 & \begin{tabular}{l|l}
0 & 100 \\
\end{tabular} & & 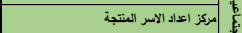 \\
\hline & 50 & 0 & 0 & 0 & & 0 & 0 & 50 & 50 & 50 & & & 5 & 50 & 50 & 50 & 50 & 50 & 100 & 0 & 0 & 0 & & & & \begin{tabular}{l|l}
0 & 100 \\
\end{tabular} & 0 & 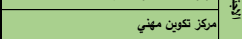 \\
\hline & 50 & 0 & 0 & & & 0 & 0 & 50 & 50 & 100 & & & & 10 & 0 & 100 & 50 & 50 & 0 & 100 & 20 & 20 & 20 & & 20 & \begin{tabular}{l|l}
0 & 100 \\
\end{tabular} & & مركز علاج طييعي اللمعاقين \\
\hline & 50 & 0 & 0 & 0 & 50 & 0 & 0 & 0 & 100 & 100 & & 0 & 0 & 10 & 0 & 100 & 50 & 50 & 0 & 100 & 0 & 0 & & 50 & 50 & \begin{tabular}{l|l}
0 & 100 \\
\end{tabular} & & 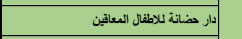 \\
\hline & 0 & 0 & 0 & 0 & & 100 & 0 & 0 & 100 & 0 & & & & & 0 & 0 & 50 & 50 & 100 & 0 & 20 & 20 & 20 & & & \begin{tabular}{|l|l|}
0 & 100 \\
\end{tabular} & & |دادزا (بشاعية \\
\hline & 50 & 0 & 0 & 0 & 50 & 0 & 0 & 0 & 100 & 0 & & 0 & 50 & 50 & 50 & 50 & 50 & 50 & 0 & 100 & 0 & 0 & 50 & 50 & 0 & \begin{tabular}{l|l}
0 & 100 \\
\end{tabular} & & لمؤسيات ثتيف فكيو \\
\hline & 100 & 0 & 0 & 0 & 0 & 0 & 33 & 33 & 34 & 100 & & 0 & 0 & 10 & 0 & 100 & 50 & 50 & 0 & 100 & 100 & 0 & & 0 & 0 & \begin{tabular}{l|l}
0 & 100 \\
\end{tabular} & & 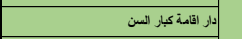 \\
\hline & 50 & 0 & 0 & & & 0 & 0 & 0 & 100 & 50 & & & & 10 & 0 & 100 & 50 & 50 & 0 & 100 & 0 & 0 & & 50 & & \begin{tabular}{l|l|}
0 & 100 \\
\end{tabular} & & حضائة الاكفال السرورين \\
\hline & 100 & 0 & 0 & 0 & 0 & 0 & 33 & 33 & 34 & 50 s $5,-5$ & & 0 & $5 t$ & 50 & 100 & 0 & 50 & 50 & 0 & 100 & 0 & 0 & 50 & 50 & 0 & \begin{tabular}{l|l}
0 & 100 \\
\end{tabular} & & 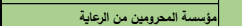 \\
\hline & & 0 & 0 & 0 & & & 33 & 33 & 34 & 100 & & & & t & 100 & 0 & 50 & 50 & 100 & 0 & 0 & 50 & 50 & 0 & \begin{tabular}{|l|l|}
0 & 10 \\
\end{tabular} & $\begin{array}{ll}00 & 0\end{array}$ & 0 & لمار مغتربين/متغبات \\
\hline & 25 & 25 & 25 & 25 & & 0 & 0 & 50 & 50 & 50 & & 1 & & 10 & 50 & 50 & 100 & 0 & 0 & 100 & 20 & 20 & 20 & & & \begin{tabular}{l|l|}
0 & 0 \\
\end{tabular} & 100 & \\
\hline & 25 & 25 & 25 & 25 & 0 & 0 & 0 & 50 & ${ }_{50}$ & 505 & & 0 & 56 & 50 & 50 & 50 & 50 & 50 & 100 & 0 & 20 & 20 & 20 & 20 & 20 & \begin{tabular}{l|l}
0 & 100 \\
\end{tabular} & & 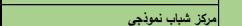 \\
\hline & 25 & 25 & 25 & 25 & & & 33 & 33 & & & & & & & 50 & 50 & 50 & 50 & 100 & 0 & 0 & 50 & 50 & & & $\begin{array}{ll}00 & 0 \\
\end{array}$ & 0 & للدن لشبابية \\
\hline & 33 & 0 & 0 & 33 & 34 & 0 & 0 & 50 & 50 & 50 & & 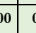 & & 50 & 100 & 0 & 50 & & 100 & 0 & 0 & 50 & 50 & 0 & \begin{tabular}{l|l}
0 & 10 \\
\end{tabular} & $\begin{array}{ll}00 & 0\end{array}$ & & مباركز التغلي والثيادات الشبابية \\
\hline & & 0 & 0 & 0 & & 0 & & 33 & 34 & & & 300 & 56 & $500-2>$ & 100 & 0 & 50 & 50 & 100 & 0 & 0 & 50 & 50 & 0 & \begin{tabular}{l|l}
0 & 10 \\
\end{tabular} & $\begin{array}{ll}00 & 0 \\
00\end{array}$ & & تزن الشباب \\
\hline & 100 & 0 & 0 & 0 & & 0 & 33 & 33 & & 100 & & & & 50 & 100 & 0 & 50 & 50 & 100 & 0 & 0 & 50 & 50 & & 0 & $\begin{array}{ll}00 & 0\end{array}$ & & بيوت الشبيب \\
\hline & 50 & 0 & 50 & 0 & 0 & 0 & 0 & 50 & 50 & 100 & & 0 & 00 & 10 & 50 & 50 & 100 & 0 & 0 & 100 & 20 & 20 & 20 & 20 & 200 & \begin{tabular}{|l|l|}
0 & 0 \\
\end{tabular} & 00 & | الثناطق المتوحة على سستوى الجهوعة \\
\hline & & 0 & 50 & 0 & & & & 50 & & & & & & & & 50 & 100 & & & 100 & 20 & & & 20 & 20 & 0 & 100 & 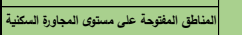 \\
\hline & 50 & 0 & 50 & 0 & & & 0 & 50 & & 50 & & & & & 50 & 50 & 50 & & 100 & 5 & 20 & 20 & 20 & 20 & 20 & \begin{tabular}{l|l}
0 & 100 \\
\end{tabular} & & 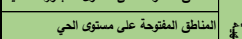 \\
\hline & 50 & $\begin{array}{c}0_{0} \\
\end{array}$ & 50 & 0 & & & 0 & 50 & 50 & 50 & & & & $50-20$ & 50 & 50 & 50 & 50 & 100 & 0 & 20 & 20 & 20 & 20 & 20 & 100 & & 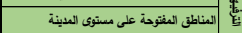 \\
\hline & 50 & 0 & 50 & 0 & & 0 & 0 & & 50 & 50 & & & & & 100 & 0 & & & & & & & & & & \begin{tabular}{l|l}
0 & 0 \\
\end{tabular} & & المناطق النقوحة على الستوى الآليليب \\
\hline 0 & & & & & & & & & & & & & & & & & & & & & & & & & & & & 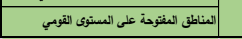 \\
\hline
\end{tabular}

المصدر : الباحثون 


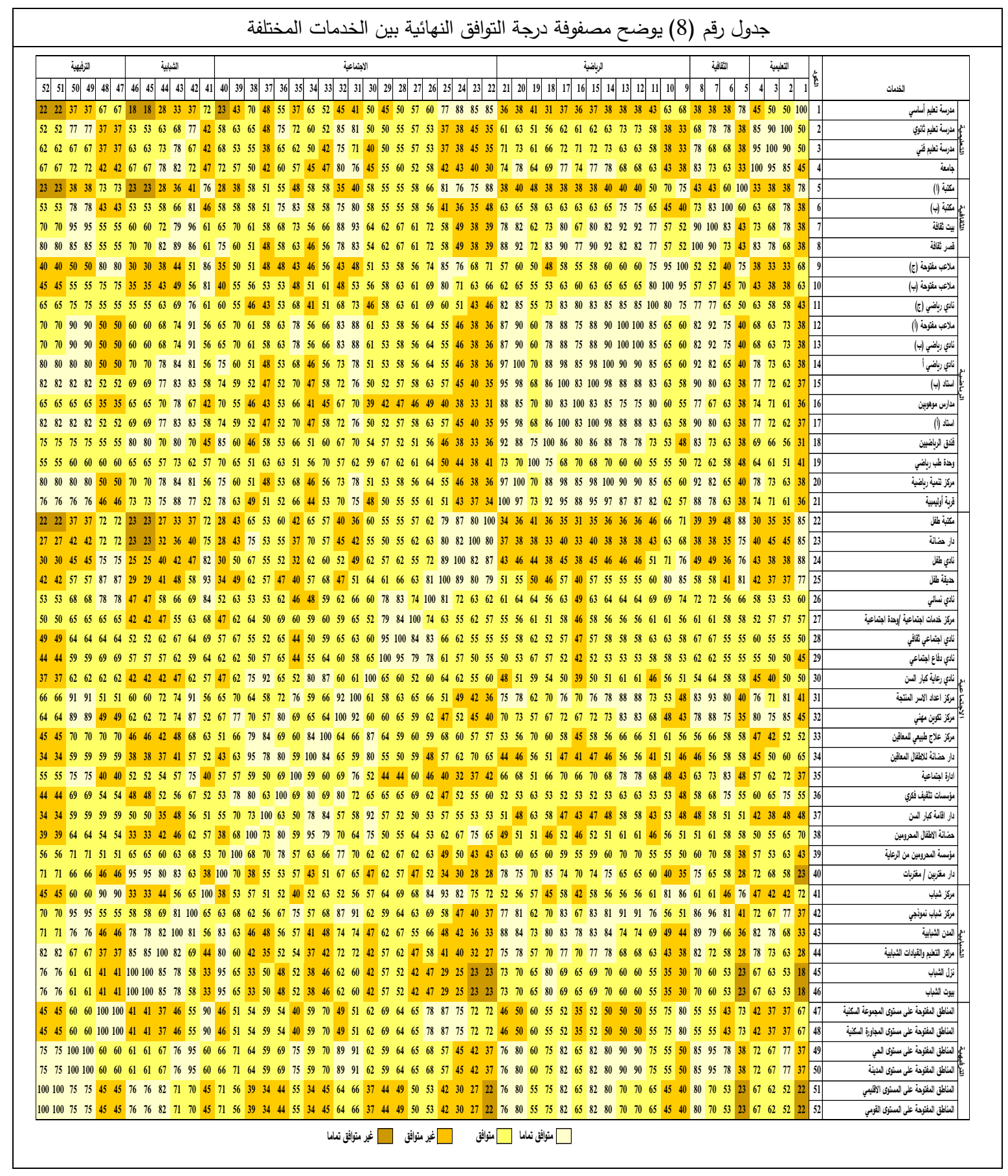


- تحديد الفراغات المبنية والمفتوحة في كل خدمة والتي تسمح بمشاركتها مع خدمات أخرى. -

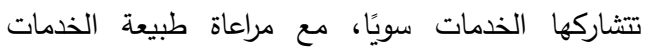
ومستواها (محلي - مركزي - إقليمي) بمعنى أن هناك ملكيات خدمات محلية قد تتشارك في فراغات خدمة إقليمية ولكن

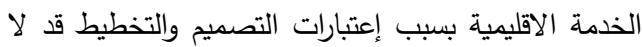

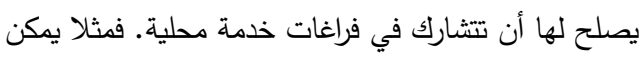

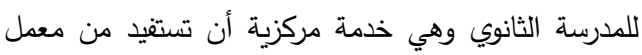
حاسب آلي في جامعة ولكن العكس لا يصلح بسبب اختلاف اشتراطات المسطحات ونصيب الفرد من الخدمة. وكمثال

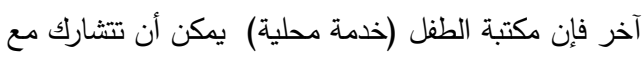

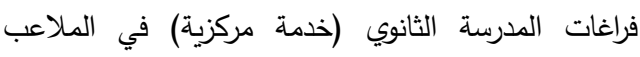
والمكتبة ولكن لا يصح العكس.

والجدول رقم (9) يوضح مصفوفة مشاركة الفراغات بين الخدمات المختلفة
وبالتالي فإن مصفوفة توافق الخدمات المختلفة تضع تصور يساعد المخطط العمراني في اتخاذ القرار بثأن الجمع بين الخدان

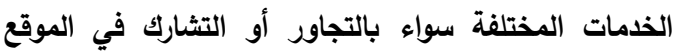
بصورة متزامنة أو متتابعة بوضع عدة بدائل يختار منهم لئه

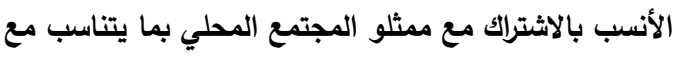

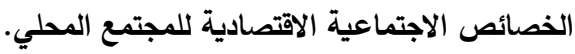

\section{3-2 مصفوفة التشارك}

تعتبر مصفوفة التشارك مصفوفة مكملة لمصفوفة التوافق بين الخدمات المختلفة حيث تقدم للمخطط العمراني صور مختلفة

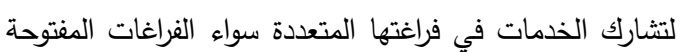
أو الفراغات المبنية. وقد تم إعداد هذة المصفوفة باتباع الخطوات التالية: رصد الفراغات المبنية والمفتوحة في كل خدمة (بالإعتماد على بيانات دلائل المعدلات والمعايير التخطيطية للخدمات بجمهورية مصر العربية من إعداد الهيئة العامة للتخطيط العمراني). 
جدول (9) مصفوفة مشاركة الفراغات بين الخدمات المختلفة

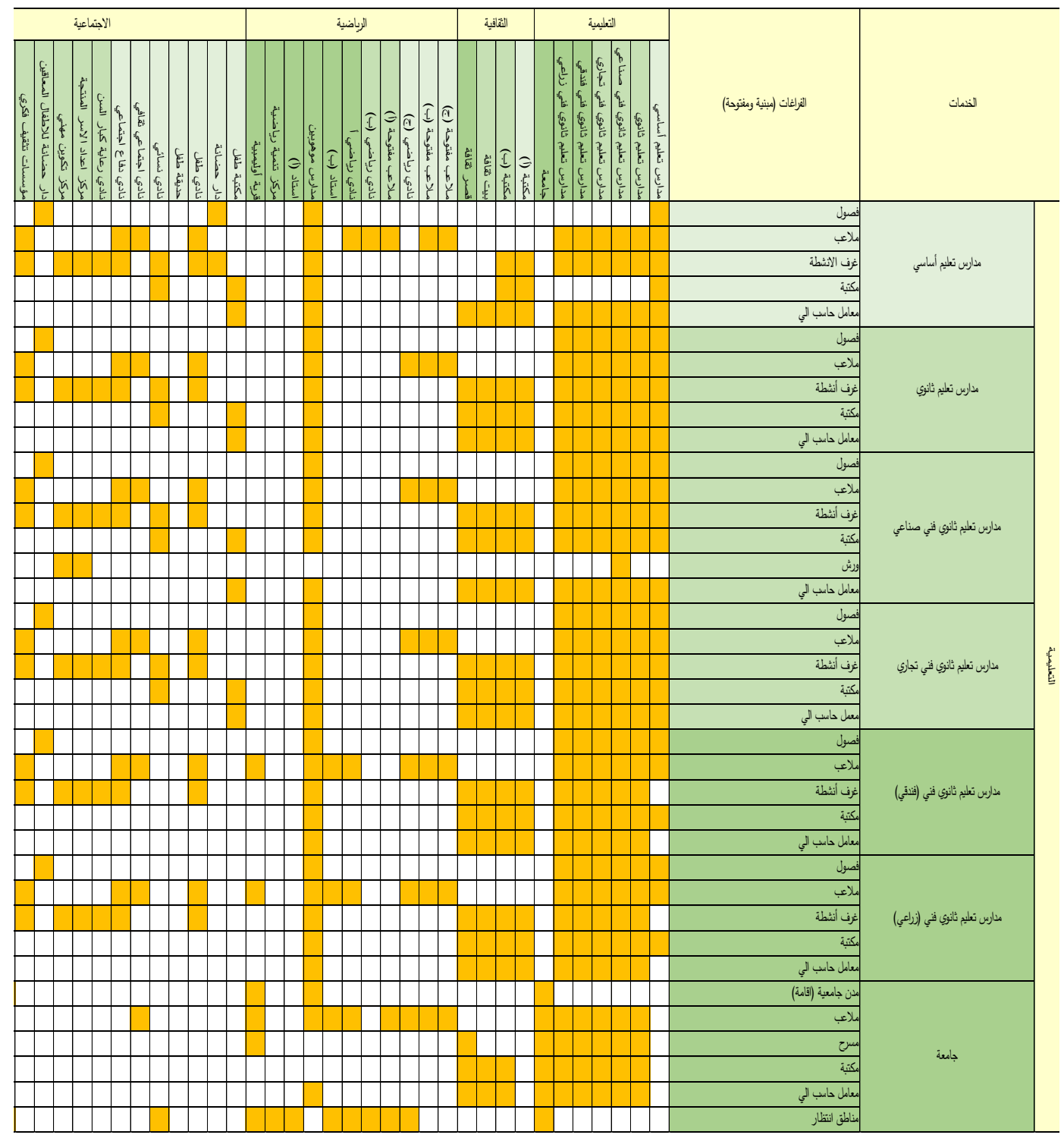


تابع جدول (9) مصفوفة مشاركة الفراغات بين الخدمات المختلفة

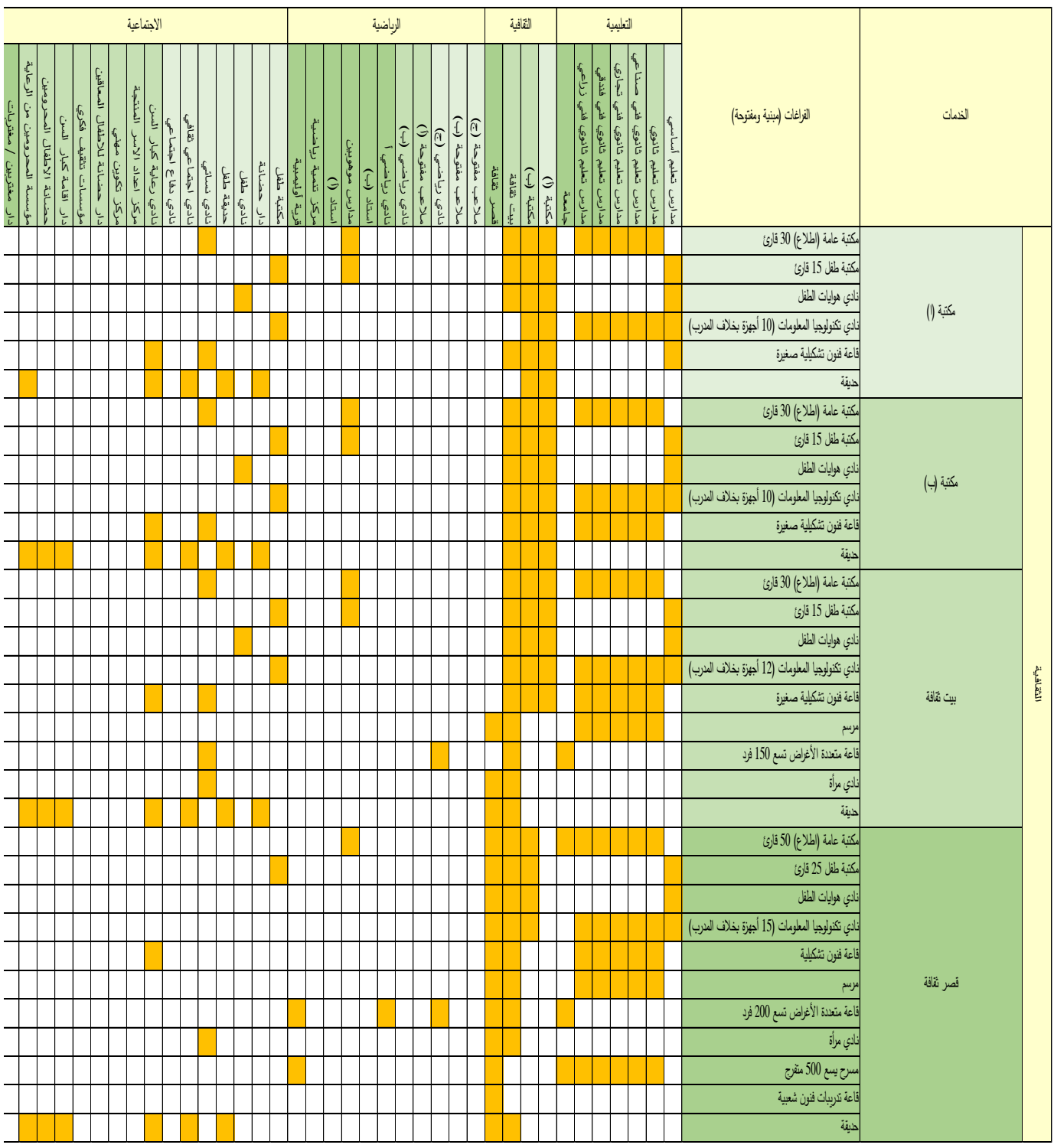


تابع جدول (9) مصفوفة مشاركة الفراغات بين الخدمات المختلفة

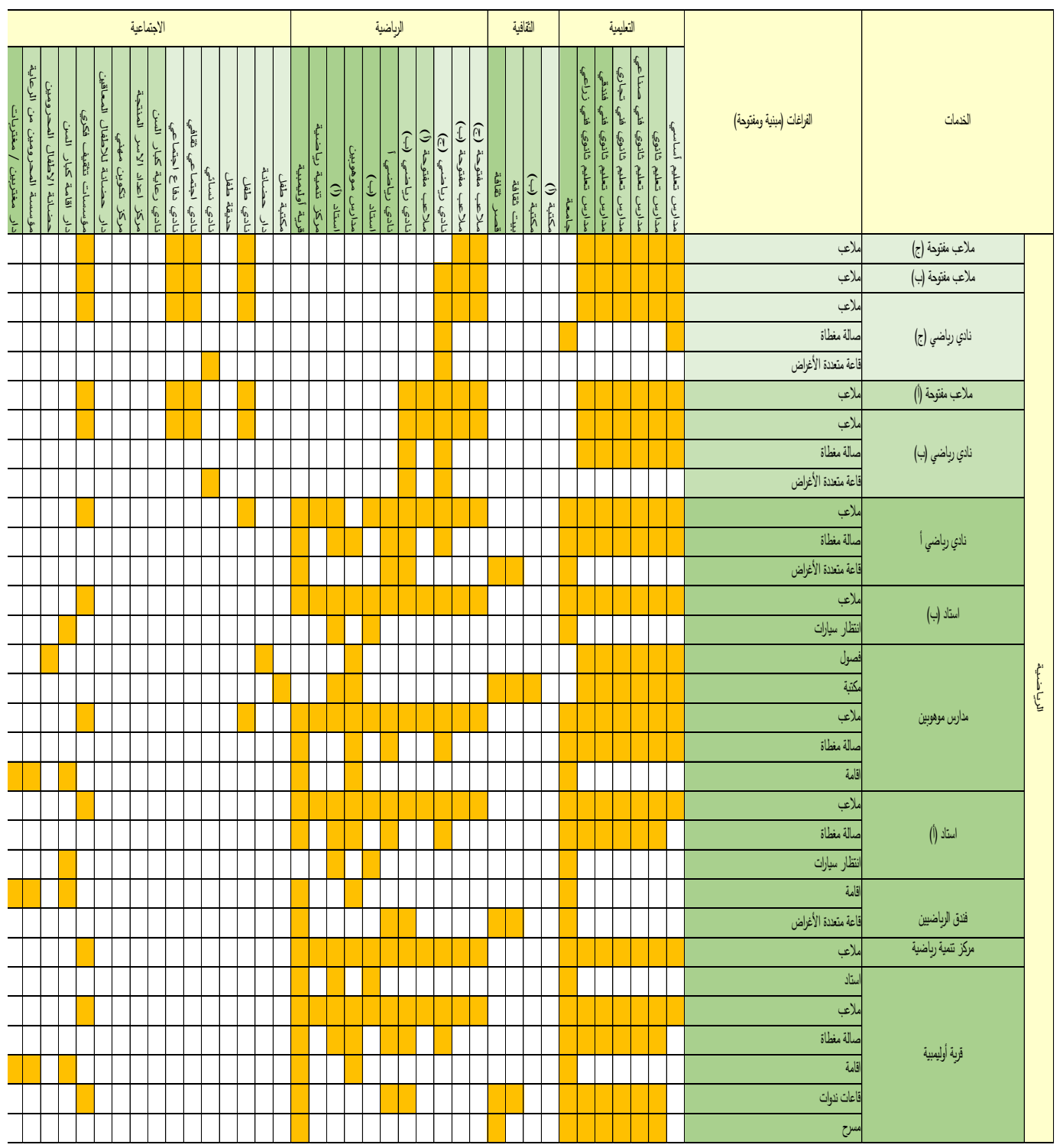


تابع جدول (9) مصفوفة مشاركة الفراغات بين الخدمات المختلفة

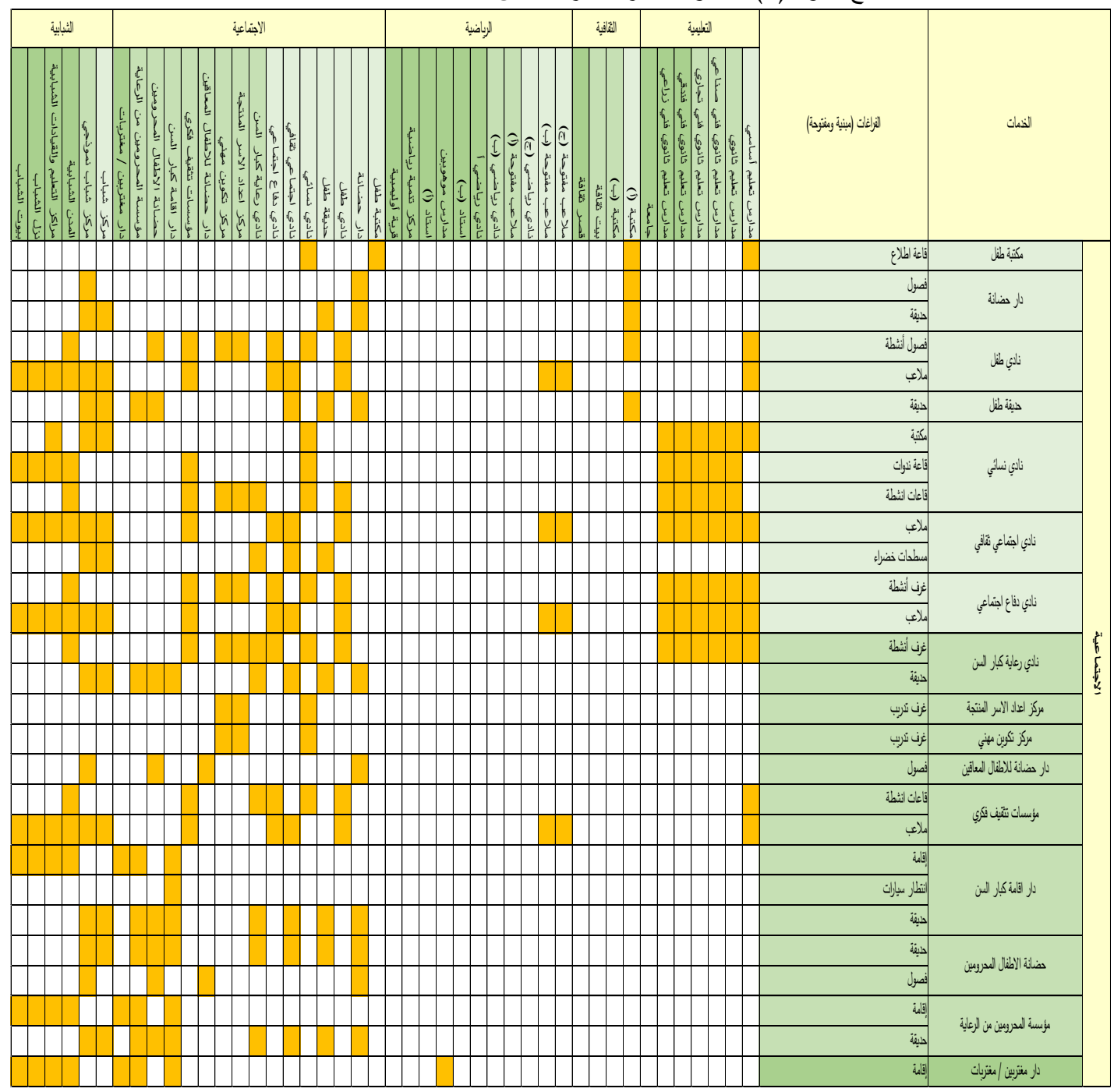


تابع جدول (9) مصفوفة مشاركة الفراغات بين الخدمات المختلفة

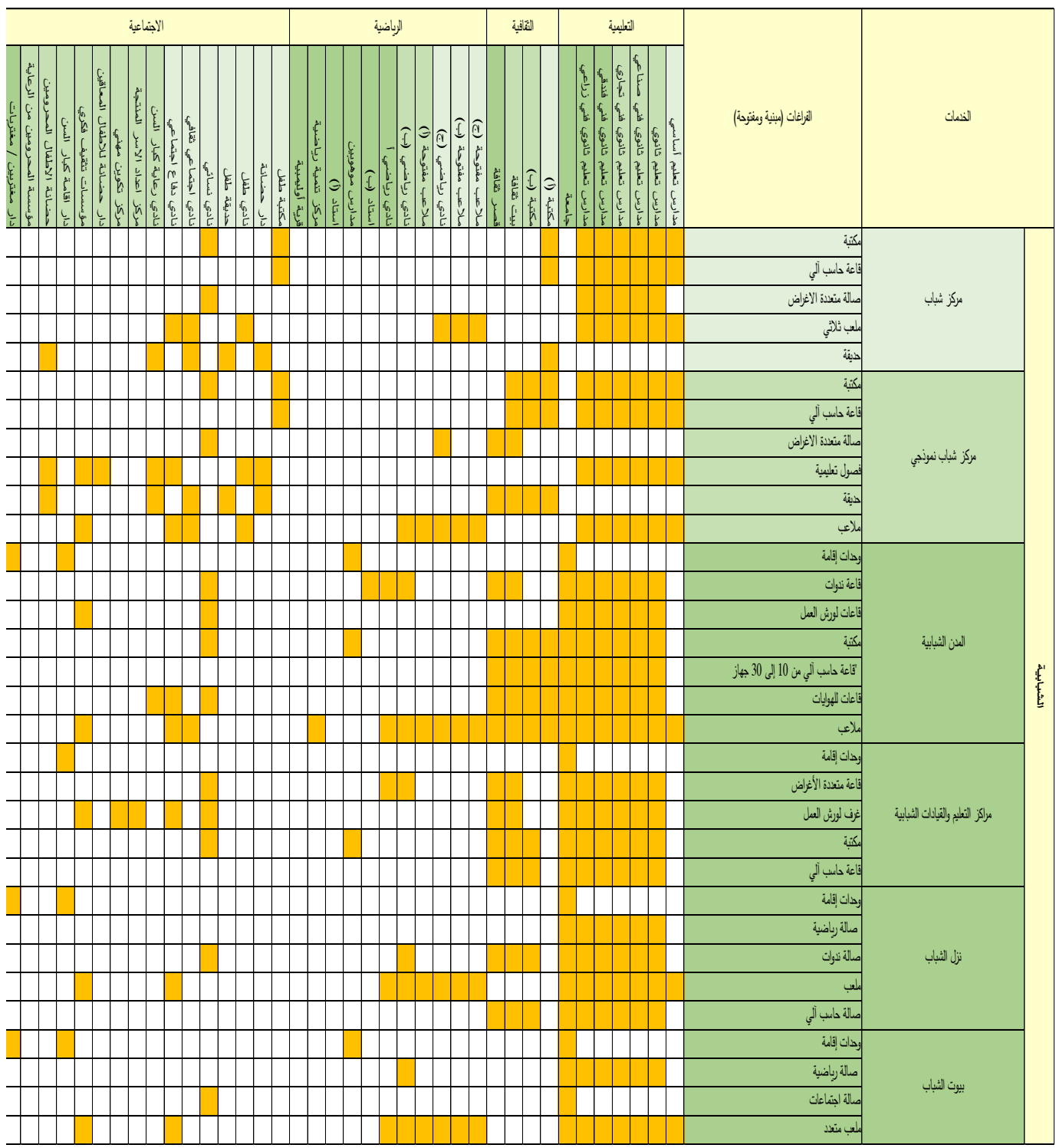


تابع جدول (9) مصفوفة مشاركة الفراغات بين الخدمات المختلفة

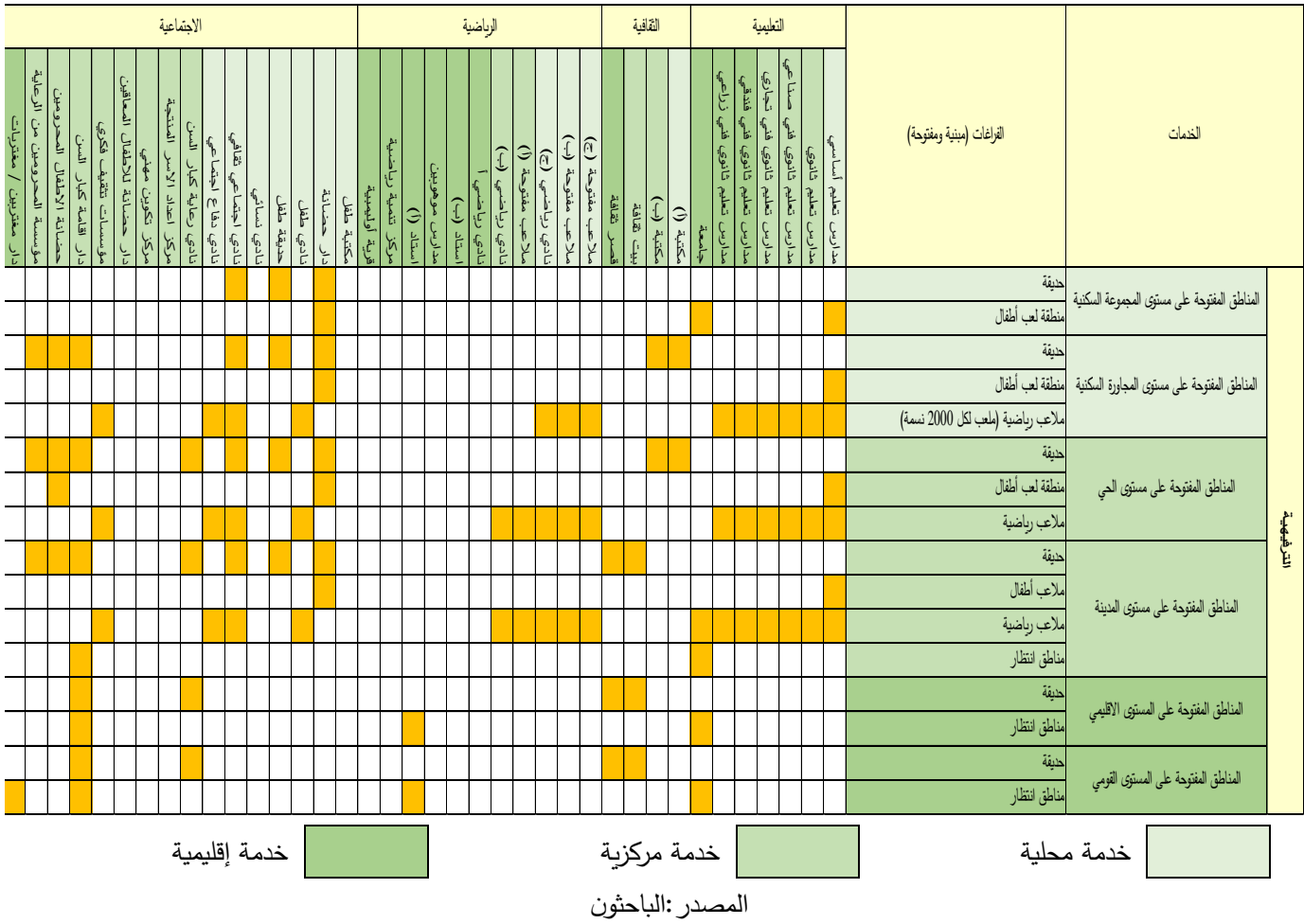

هناك فرق بين كل من المستوى المكاني الجزئي والمستوى

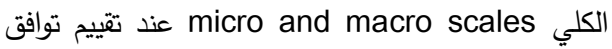
استخدام الأراضي، حيث لا يمكن استخدام العلاقات

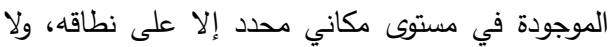
يمكن استخدامها لاستتتاج وجود تأثيرات خارجية مماثلة على مستويات مكانية أخرى.

يوصي البحث صانعي القرار بأخذ توافق استخدام الأراضي في الاعتبار عند تطبيق سياسة الاستخدام المختلط للأراضي من أجل تقليل العوامل الخارجية السلبية وخلق مناطق حضرية أكثر استدامة. التوافق بين الاستخدامات المتشاركة في نفس الموقع

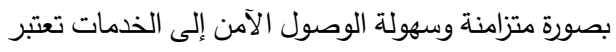

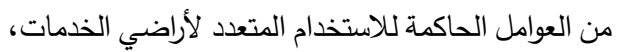
ولذا فقد تم التوصل إلى مصفوفة التوافق بين الخدمات
4- النتائج والتوصيات قد يلعب الاستخدام المختلط للأراضي دورًا كبيرًا في حل فلتوفيات المشكلات الحضرية مثل التلوث والإفراط في استهلاك دلك

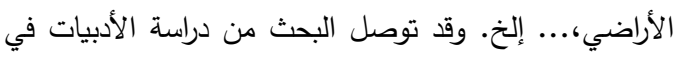

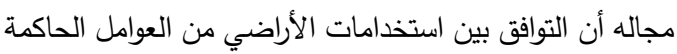

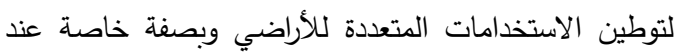
توطين الخدمات المجتمعية، ومن ثم يجب تضمين دراسة التوافق

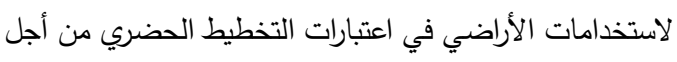

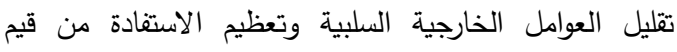
الأراضي، و بالتالي خلق مدن أكثر قيمة وأكثر أداءً وأكثر استدامة. وقد خلص البحث للنتائج التالية:

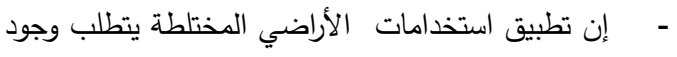

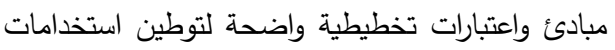

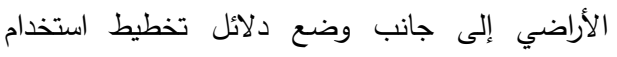
الأراضي التي تراعي التوافق بين الاستخدمات المختلفة. 
الصيانة الدورية لمباني مشروعات الاستخدام المتعدد

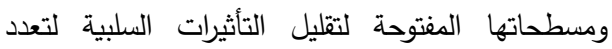
الاستخدام على استهلاك الموارد.

\section{المراجع}

م. ك. مدبولي، "تطوير منظومة جديدة للعمران [1]

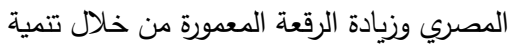
الظهير الصحراوى للقري والمدن المصرية،" تأليف المؤتمر العربي الاقليهي "الترابط بين ولين

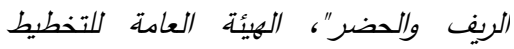

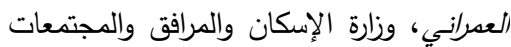
العمرانية الجديدة، 2005.

جهاز شئون البيئة، "تقرير حالة البيئة في مصر [2006،

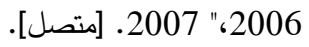

Available:

http://www.eeaa.gov.eg/porta 1s/0/eeaaReports/SoE2007Ar/ 09-urban.pdf

.

إ. م. إبراهيم، المخططات التنصيلية للمدن [3]

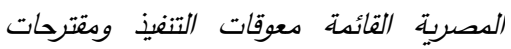
الحلول، كلية التخطيط العمراني والإقليمي، جامعة القاهرة،: رسالة ماجستير غير منشورة، .2016

م. ط. م. سليم، "، ضبط التعارض بين قانون [4]

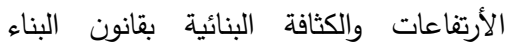
المصري الموحد رقم 119 لسنة 2008،" النشرة

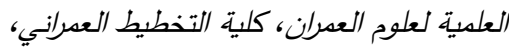

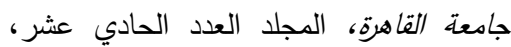
.2008

و.زارة الاسكان والمرافق والمجتمعات العمرانية، [5]

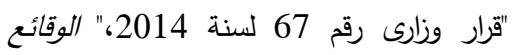
المصرية ، 9 فبراير 2014.
المختلفة لتكون أداة داعمة لتطبيق أسلوب الاستخدام المتعدد للأراضي في مصر • لتحديد درجة التوافق بين استخدامات الأراضي الحضرية،

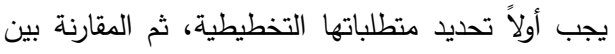
خصائص كل نوع منها ودراسة التأثيرات والعلاقات بينها. متغيرات حساب درجة التوافق بين الخدمات في مصر تضم (مستوى الخدمة - الفئة العمرية المستهدفة - طبيعة لئل

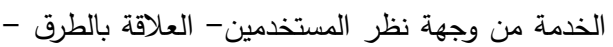
سهولة الوصول - الحاجة للهدوء- الحاجة إلى الأمان توليد حركة مرورية - التأثيرات البيئية للخدمة)

توصل البحث إلى مصفوفة توافق الخدمات المختلفة (الحالة المصرية)، وهي تضع تصور يساعد المخطط

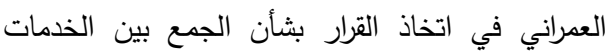
المختلفة سواء بالتجاور أو التشارك في الموقع بصورة متزامنة أو متتابعة بوضع عدة بدائل يختار منهم الأنسب

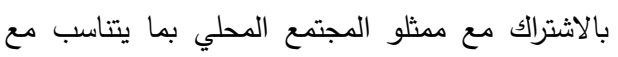

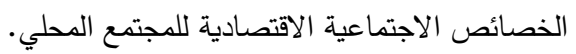
استكمالًا لمصفوفة التوافق تم إعداد مصفوفة التشارك بين الخدمات المختلفة حيث تقدم للمخطط العمراني صور مختلفة لتثارك الخدمات في فراغاتها المتعددة سواء المفتوحة أو المبنية.

يعتمد تحديد عناصر الخدمة (الفراغات) التي يمكن أن تتشاركها الخدمات سويًا، على طبيعة الخدمات ومستواها (محلي - مركزي - إقليمي) بمعنى أن هناك خدمات

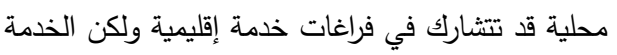

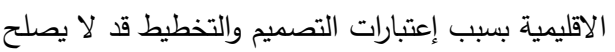
لها أن تتشارك في فراغات خدمة محلية. - سلامة المباني وتوافر المسطحات اللازمة لتوطن

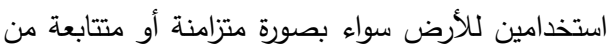

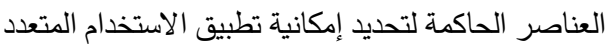
لنأراضي. 
Design, vol. 38, no. 3, pp. 388-410, 2011.

[15] Masoomi, Z., Mesgari, M. S., \& Hamrah, M., "Masoomi, Z., MesgariAllocation of urban land uses by Multi-Objective Particle Swarm Optimization algorithm," International Journal of Geographical Information Science, vol. 27, no. 3, pp. 542-566, 2013.

[16] Taleai, M., Sharifi, A., Sliuzas, R., \& Mesgari, M., "Taleai, M., Sharifi, A., Sliuzas, R., \& MesEvaluating the compatibility of multifunctional and intensive urban land uses," International Journal of Applied Earth Observations and Geoinformation, vol. 9, no. 4, pp. 375-391, 2007.

[17] T. I. f. L. Government, "Understanding the Basics of LAND USE AND PLANNING: Glossary of Land Use and Planning Terms," The Institute for Local Government, California, U.S.A, 2010.

[18] M. o. t. Environment, "Ministry of the Environment, Conservation and Parks Guidelines, Canada. Available at: https://www.ontario.ca/page/d-1-3land-use-compatibility-definitions. Updated: June 5, 2019, Published: April 14, 2016," 5 June 2019. [Online]. Available: Ministry of the Environment, Conservation and Parks

Guidelines,https://www.ontario.ca/ page/d-1-3-land-use-compatibilitydefinitions. [Accessed 25 January 2020].

[19] Rusek, R., Marsal-Llacuna, M. L., Fontbona, F. T., \& Llinas, J. C., "Compatibility of municipal services based on service similarity," Cities, vol. 59, pp. 4047, 2016.
[6] Song, Y., \& Knaap, G. J. , "Measuring the effects of mixed land uses on housing values," Regional Science and Urban Economics, vol. 34, no. 6, pp. 663680, 2004.

[7] A. P. Association, "The principles of smart development," American Planning Association., Chicago, IL, 1998.

[8] C. f. t. N. Urbanism, "Charter of the new urbanism," Bulletin of Science, Technology \& Society, vol. 20, no. 4, pp. 339-341, 2000.

[9] Habitat, "A new strategy of sustainable neighbourhood planning: Five principles," United Nations Human Settlements Programme., Nairobi, Kenya, 2014.

[10] Schwanke, D., \& Flynn, T., Mixeduse development handbook, Washington, DC: Urban Land Institute., 2003.

[11] Hoppenbrouwer, E., \& Louw, E., "Hoppenbrouwer, E Mixed-use development: Theory and practice in Amsterdam's Eastern Docklands," European Planning Studies, vol. 13, no. 7, pp. 967-983, 2005.

[12] P. Wardner, "Explaining mixed-use developments: A critical realist's perspective," in 20th Annual Pacific-Rim Real Estate Society Conference, New Zealand, 2014.

[13] J. D. Herndon, Mixed-Use Development in Theory and Practice: Learning from Atlanta's Mixed Experiences., Georgia: Georgia Institute of Technology, 2011.

[14] Haque, A., \& Asami, Y., "Optimizing urban land-use allocation: case study of Dhanmondi Residential Area, Dhaka, Bangladesh. ," Environment and Planning B: Planning and 
[25] Rusek, R., Colomer Llinas, J., \& Melendez Frigola, J., "Rusek, R., Colomer Llinas, J., \& MelendDecision support framework for space-use efficiency and arrangement of public services," Rusek, R., Colomer Llinas, J., \& Melendez Frigola, J. (2020). Decision support framework for space-use efJournal of Urban Planning and Development, vol. 146, no. 1, 2020.

[26] F. C. '. Jr., Urban Land use Planning, Urbana: University of Illinois, 1965.
[20] S. \&. S. T. Eom, "Spatial distribution of pedestrian space in central Tokyo.," Eom, S., \& Suzuki, T. (2019). Spatial distribution of pedestriaInternational Review for Spatial Planning and Sustainable Development, vol. 7, no. 2, pp. 108124, 2019.

[21] Abedini, A., Lotfian, M., \& Moradi, M., "Land Use Compatibility Assessment Using a Modified Topsis Model: a Case Study of Elementary Schools in Tehran," The International Archives of Photogrammetry, Remote Sensing and Spatial Information Sciences, 40, vol. 40, no. 1, p. 5, 2015.

[22] Vaezi, S., Mesgari, M., \& Kaviary, F., "Evaluation of Effecting Parameters on Optimum Arrangement of Urban Land Uses and Assessment of Their Compatibility Using Adjacency Matrix.," The International Archives of Photogrammetry, Remote Sensing and Spatial Information Sciencec, vol. 40, no. 1, p. 725, 2015.

[23] townofunion, "townofunion.com," [Online]. Available: http://www.townofunion.com/pdfs/ Comprehensive\%20Plan\%20Tech $\%$ 20Reports/TBR\%20Chapter\%20 6\%20Land\%20Use $\% 20$ Compat $\% 2$ 0Bound\%20Version\%201-1309.pdf. [Accessed 4 April 2019].

[24] Cheniki, K., Baziz, A., \& Boudiaf, B., "CheniEvaluating Relationship between Mixed-land Use and Landuse Compatibility in Algiers Bay," Cheniki, K., Baziz, A., \& Boudiaf, B. (2019). Evaluating Relationship between Mixed-land

Use and Land-usInternational Journal of Environmental and Science Education-IJESE, vol. 14, no. 7, pp. 389-404, 2019. 


\section{A mechanism for public services allocation in Egypt, Depending on their compatibility}

The concept of mixed land uses emerged because of what cities have suffered because of the problems of segregation in land use, such as urban sprawl, the high cost of transportation to workplaces and services, and environmental pollution. Due to the many problems that cities face, especially the Egyptian ones, such as urban sprawl on agricultural lands, and the inefficiency of service provision, in addition to The difficulty of implementing detailed plans for cities because of the lack of state-owned land suitable for urban development, which corresponds to the areas required to provide the cities' current and future needs for services, so the multiple use of land can be an appropriate solution to overcome the problem of availability of land as well as to achieve maximum benefit from the value of land. The research problem is based on the absence of a current mechanism by which compatibility between services can be determined, and to decide their simultaneous or sequential coexistence in the same site within the context of multiple use of lands. The research deals with this problem by defining the main objective of the research, which is to uncover the theoretical framework that includes the current problems of Egyptian urbanization, then review and analyze the literature of the application of mixed land uses, as well as the compatibility between land uses and analyze previous studies that dealt with calculating the compatibility between land uses in order to determine the variables of calculating the degree of compatibility Between services in Egypt, and the preparation of a compatibility matrix between services in Egypt to assist the urban planners and decision-makers in identifying alternatives to services that are suitable for simultaneous or consecutive joint coexistence, as well as a participation matrix explaining the spaces that can be provided by each service to share with other services, whether similar or different service. 